\title{
MEDIÇÃO DA EVOLUÇÃO DA PRODUTIVIDADE TOTAL DOS FACTORES: O ÍNDICE DE MALMQUIST**
}

\author{
João Rebelo**
}

\begin{abstract}
Dependendo a competitividade de um país do valor e crescimento sustentado da produtividade das organizações económicas que integram a sua economia, para complemento da informação proporcionada por indicadores parciais de performance, é de grande importância conseguirse um indicador capaz de captar as fontes e aferir de forma sintética, objectiva e adequada a evolução da produtividade das unidades como um todo. Este indicador é o índice de produtividade total dos factores (PTF), susceptível de ser quantificado através dos números índices de Malmquist, Fischer ou Törnqvist, prestando-se neste texto especial atenção ao primeiro. $O$ índice PTF de Malmquist é calculado com base em funções distância em relação à função fronteira, recorrendo, para a estimação desta, a métodos deterministas não paramétricos (DEA) ou a funções estocásticas paramétricas (AFE). Para ilustrarmos o processo de cálculo, aplicamos os dois métodos a um panel equilibrado de 26 explorações agrícolas localizadas no Planalto Mirandês para o período 1990-97.
\end{abstract}

Palavras-chave: Produtividade, factores, números índices, Malmquist.

\section{ENQUADRAMENTO/JUSTIFICAÇÃO DO TEMA}

Perante a crescente globalização dos mercados e liberalização das trocas, a competitividade do sistema económico é uma preocupação central de

** Universidade de Trás-os-Montes e Alto Douro; Docente Convidado do Instituto Universitário de Desenvolvimento e Promoção Social, Pólo de Viseu da Universidade Católica Portuguesa. 
qualquer nação. Para Porter (1990), a competitividade de um País apoia-se na produtividade, sendo o valor desta e o seu contínuo crescimento, no longo prazo, os principais determinantes do nível de bem estar da população. Este autor considera a produtividade como sendo o valor da produção por unidade de capital ou trabalho, dependendo o valor da mesma tanto da qualidade e características do produto (que determinam o seu preço) como da eficiência com que são produzidos. $\mathrm{O}$ crescimento sustentado da produtividade exige que a economia se "aprimore a si mesma", devendo as empresas melhorar de forma implacável a produtividade dos sectores, adicionando características desejáveis aos bens, desenvolvendo novas tecnologias ou impulsionando a eficiência da produção ${ }^{1}$. Isto é, dependendo a competitividade de um país do crescimento sustentado da produtividade das organizações económicas que o integram, para a definição de estratégias conducentes à deslocação das "fronteiras de produção" e ao "eficaz aproveitamento dos recursos disponíveis", é de grande importância saber a origem e aferir correctamente a evolução da produtividade total dos factores ${ }^{2}$ implicados na actividade das unidades produtivas (sejam elas de fins lucrativos ou não). Tanto ao nível da economia, como de cada sector, nem sempre é fácil atingir aquele desiderato, desempenhando os números índices ${ }^{3}$ papel de relevo na avaliação objectiva da performance da economia e das organizações produtivas que a integram.

\subsection{Complexidade da "moderna economia" e índices deperformance económica}

Estatisticamente e a um nível agregado tem aumentado a complexidade de medição da evolução da performance das economias, devido ao efeito simultâneo e conjugado de três poderosas forças:

??Globalização. A crescente proporção nas trocas, no investimento e no valor do crédito de origem/destino extra nacional, assim como o aumento de fluxos de turistas e de trabalhadores estrangeiros originam economias, cada vez mais globalizadas ${ }^{4}$, complicando a quantificação do que é performance nacional.

??Invisíveis. Tradicionalmente, as estatísticas estavam concebidas para a recolha de informação sobre a produção de bens físicos. No entanto, é crescente a proporção no produto interno bruto (PIB) de produtos que não são bens físicos, menos visíveis, logo de mais difícil quantificação. Em sectores com grande importância na economia dos países mais avançados, como sejam os ligados aos sistemas de conhecimento, informação e comunicação, serviços financeiros, 
educação, saúde, serviços judiciais, não é fácil definir e quantificar o produto, logo a produtividade. Por exemplo, em campos como a saúde ou a educação, se considerarmos o produto como sendo o número de horas de trabalho, certamente que a produtividade não aumenta em termos de tempo despendido. A alteração na diversidade de bens e serviços transaccionados, em cada momento, torna difícil e complexa a tarefa de definição de indicadores objectivos e comparáveis no tempo e espaço.

??Tecnologia. Novos bens, produtos com curtos ciclos de vida e rápidas melhorias na qualidade tornam complicado medir alterações na quantidade produzida e nos preços. Os preços dos bens e serviços mudam, frequentemente, em resposta a alterações tecnológicas e outros factores condicionantes do custo de produção e da qualidade, o que conduz a alterações dos padrões de compra do consumidor, ou seja, a permanente modificação dos cabazes de bens representativos da procura incluídos, tradicionalmente, no cálculo dos índices de preços do consumidor (IPC).

Perante a globalização, as constantes e rápidas mudanças tecnológicas e a expansão da chamada "nova economia", os indicadores estatísticos tradicionais (e.g. o conhecido IPC baseado no índice de Laspeyres) têm dificuldade em captar e medir adequadamente as alterações ocorridas no sistema económico de determinado país ou região. Partindo do possível erro de medição do IPC adoptado nos Estados Unidos da América (com sobreestimação da taxa de inflação em cerca de 1,1\%/ano, entre 1960 e 1995), detectado pela Comissão liderada por M. Boskin (Boskin et al., 1997) e das respectivas consequências nefastas sobre indicadores económicos como o crescimento do PIB, a produtividade do trabalho e o défice orçamental, em The Economist (November 23rd, 1996: 86) refere-se: "Estatísticos defrontam-se com o grande desafio de tentarem eles próprios elevarem a qualidade da sua própria produção. Há certamente lugar para melhoria. Todavia, é provavelmente um inevitável, ainda que irónico, facto que na chamada época da informação, quando super-computadores conseguem manipular montanhas de dados e satélites conseguem captar os movimentos de pessoas e máquinas, o conhecimento das pessoas acerca da evolução da economia seja menos exacto que o era anteriormente". 


\subsection{Indicadores de desempenho das organizações produtivas}

A nível microeconómico a avaliação do desempenho das unidades produtivas é, igualmente, um processo complexo, podendo recorrer-se a uma diversidade de indicadores relacionados com: medidas de performance técnica (aspectos técnicos da produção) e de qualidade (e.g. disponibilidade e tipo dos factores produtivos utilizados, grau de aceitação no mercado dos produtos transformados); indicadores financeiros (e.g. rendibilidade do activo, do capital próprio ou outros rácios); e medidas de performance física, como sejam a produtividade parcial dos factores produtivos.

Este conjunto de indicadores desempenham um papel interessante na captação de informação sobre a gestão das empresas, permitindo detectar forças e fraquezas subjacentes à sua estrutura, organização e funcionamento. Todavia, perante a complexidade das envolventes externa e interna da maioria das organizações, uma gestão eficaz requer um indicador capaz de proporcionar ensinamentos válidos sobre a origem e a evolução da performance da unidade produtiva como um todo. Esta medida é a produtividade total dos factores (PTF), sendo o conhecimento das principais fontes subjacentes à sua evolução de grande importância para o estabelecimento de estratégias conducentes à adequada afectação de recursos. Na secção seguinte apresentamos os principais números índices susceptíveis de utilizar na medição da PTF.

\section{MEDIÇÃO DA PRODUTIVIDADE TOTAL DOS FACTORES ATRAVÉS DE NÚMEROS ÍNDICES}

A medição da evolução da PTF pode ser feita recorrendo aos índices quantidade (Q) de Fischer (1922), de Törnqvist (1936) ou de Malmquist (1953), uma vez que os três, genericamente, preenchem as propriedades ${ }^{5}$ exigidas pela teoria económica que está subjacente à definição e construção dos números índices (Coelli et al., 1998: cap. 4).

\subsection{Os índices quantidade de Fischer e de Törnqvist}

Para uma breve apreciação dos índices Fischer e de Törnqvist, consideremos que: $\mathrm{p}_{\mathrm{ij}}$ e $\mathrm{q}_{\mathrm{ij}}$ representam, respectivamente, o preço e a quantidade do iésimo bem $(i=1,2, \ldots, N)$ no jésimo período $(j=s, t)^{6}$; as quantidades e os preços podem referir-se tanto a produtos como a factores; $\mathrm{s}$ 
é o "período base ou de referência" e t o "período corrente", em relação ao qual o índice se refere e é calculado.

$\mathrm{O}$ índice quantidade de Fischer $\left(\mathrm{Q}_{\mathrm{st}}{ }^{\mathrm{F}}\right)$ é a média geométrica dos índices quantidade de Laspeyres $\left(\mathrm{Q}_{\mathrm{st}}{ }^{\mathrm{L}}\right)$ e de Paasche $\left(\mathrm{Q}_{\mathrm{st}}{ }^{\mathrm{P}}\right)$, com:

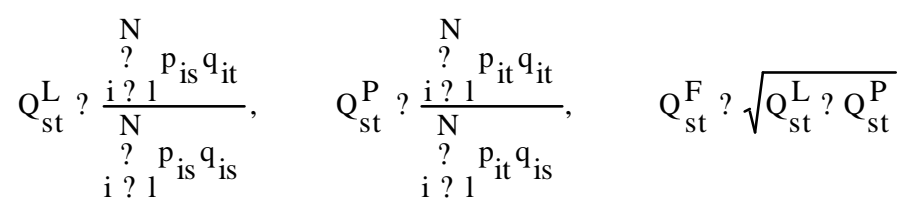

O índice quantidade de Törnqvist é a média geométrica ponderada do rácio das quantidades, sendo as ponderações dadas pela média aritmética do valor das shares (pesos relativos) nos períodos $\mathrm{s}$ e t. Nas formas multiplicativa e aditiva (variações na forma logarítmica) este índice é dado por:

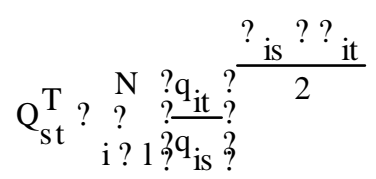

$\mathrm{ou}$

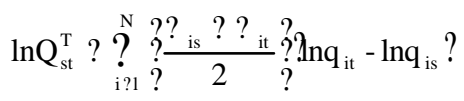

sendo ? is ? $\mathrm{p}_{\text {is }} \mathrm{q}_{\text {is }} / ?_{\text {i? } 1}^{N} \mathrm{p}_{\text {is }} \mathrm{q}_{\text {is }}$ o peso relativo (share) do iésimo bem no conjunto dos bens, no período base s (cálculo semelhante pode ser feito para t). Por facilidade computacional, o índice quantidade de Törnqvist é por norma calculado tendo por base a forma logarítmica.

Na prática (Coelli et al, 1998: 81), "o índíce de Törnqvist parece ser o preferido, sendo o índice de Fischer recomendado apenas para situações em que é requerida a propriedade adicional da auto-dualidade ou quando existem zeros nos dados". De qualquer modo, em ambos os métodos e para casos concretos, o cálculo da evolução da PTF entre os períodos $s$ e $t$ é um processo executado em duas fases. Na primeira, calculam-se os respectivos índices quantidade de produtos e de factores aplicando (1) ou (2). $\mathrm{Na}$ segunda calculam-se e comparam-se os rácios entre os índices quantidade de produtos e de factores determinados na fase anterior. 
A popularidade dos índices de Fischer e Törnqvist resulta fundamentalmente (Griffel-Tatjé e Lovell, 1996) de ambos: poderem ser calculados a partir de dados observados sobre quantidades e preços, sem necessidade de estimar a fronteira produtiva ${ }^{7}$; serem compatíveis com representações flexíveis da fronteira, isto é, são índices "superlativos".

Relativamente aos índices de Törnqvist e Fischer, o índice de Malmquist apresenta as seguintes vantagens (Griffel e Lovell, 1996): primeira, não exige a adopção da hipótese de maximização do lucro ou minimização do custo, o que é particularmente relevante para as situações em que se está analisar o sector público ou organizações não lucrativas, nas quais, com frequência, não se verifica tal comportamento; segunda, não necessita de informação sobre preços dos produtos e factores, a qual frequentemente surge distorcida ou é mesmo inexistente. Finalmente, permite a decomposição da evolução da PTF em modificações da eficiência produtiva (aproximação ou afastamento em relação à fronteira de produção, efeito catching-up) e em alterações tecnológicas (movimentos da própria fronteira produtiva).

A principal fraqueza do índice de Malmquist está no facto de apenas ser aplicável na presença de panel data, enquanto os primeiros podem ser calculados com apenas uma observação em cada período.

Perante as suas vantagens, o índice de Malmquist constitui o veículo natural de quantificação da alteração da PTF, havendo, porém, situações ${ }^{9}$ em que o seu valor coincide com o de Törnqvist ou de Fischer.

\subsection{O índice PTF de Malmquist}

Como o índice de Malmquist é calculado a partir de medidas de distância em relação à função fronteira, começamos por fazer uma breve descrição do conceito fronteira e respectivos métodos de estimação, após o que descrevemos a metodologia de cálculo do índice PTF de Malmquist.

\subsubsection{Funções fronteira e métodos de estimação}

Em qualquer manual de microeconomia, a função de produção vem definida como sendo, para certo estado do conhecimento tecnológico, o máximo produto que é susceptível de obter com determinado conjunto de factores produtivos. De forma semelhante, a função custo representa o mínimo custo suportado para a obtenção de certo nível de produção, dados os preços dos factores. Por último, a função lucro representa o máximo lucro 
susceptível de atingir, dados os vectores dos preços dos produtos e dos factores.

Nas definições anteriores, as palavras máximo e mínimo são importantes, podendo, a qualquer dos casos, ser aplicada a noção de fronteira. Para um conjunto de observações, a fronteira estimada indica que não é possível encontrar nenhuma dbservação acima (no caso das funções de produção e lucro) ou abaixo da mesma (no caso da função custo).

A diferença, positiva ou negativa, entre o valor fronteira estimado para a produção, lucro ou custo e o da respectiva observação pode interpretar-se como medida de ineficiência produtiva. Quando o óptimo é definido pela função de produção, a medida de eficiência obtida designa-se de eficiência técnica. Se, pelo contrário, a comparação se realiza considerando um óptimo definido em termos de objectivo económico visado pelas empresas (minimização do custo, maximização do rendimento ou do lucro), a medida de eficiência obtida denomina-se de eficiência económica.

A medição da ineficiência produtiva é o principal motivo para a estimação das funções fronteira. A utilização de métodos de regressão tradicionais, baseados no pressuposto de que o termo erro aleatório segue uma distribuição de probabilidade bicaudal de média zero e variância homocedástica, conduz ao ajustamento de modelos de comportamento em que os valores estimados traduzem a experiência "média" das empresas observadas, possuindo estas uma estrutura tecnológica de transformação comum. Estes métodos são razoáveis se estamos apenas interessados no conhecimento das propriedades económicas da tecnologia média da indústria. Pelo contrário se o interesse incidir nos aspectos de tecnologia de "ponta" ou de "melhor prática" da indústria, então tem de adoptar-se uma estrutura do erro que permita que algumas empresas sejam mais eficientes que outras. Decisões erradas dos gestores, regulamentações governamentais, barreiras à entrada e saída do sector e imperfeições do mercado são elementos que contribuem para o aparecimento de ineficiência produtiva.

Os métodos de medição da eficiência produtiva diferem pela forma como:

??é especificada a fronteira, por funções paramétricas ou não paramétricas;

??é construída a fronteira, através de técnicas de programação matemática ou estatísticas;

??são interpretados, em relação à fronteira, os desvios, representando estes apenas ineficiência ou uma combinação desta com factores aleatórios fora do controlo da empresa, tendo-se funções fronteira deterministas (não permitem observações acima da fronteira, no caso da função de produção ou lucro, ou abaixo da fronteira, para o caso da 
função custo) ou estocásticas (permitem que algumas observações se localizem acima ou abaixo da fronteira, devido a causas aleatórias).

Forsund et al. (1980), Rebelo (1992) e Pastor (1995) são alguns dos autores que proporcionam revisões bastante claras sobre os diferentes modelos-fronteira, ao nível da especificação e estimação da fronteira, assim como das vantagens e inconvenientes dos respectivos métodos. Em casos concretos, a escolha de um dos métodos deve basear-se em informações sobre a qualidade dos dados, na forma como foram gerados e, acima de tudo, nos objectivos do estudo. Além disso, em caso de dúvida, de modo a avaliar-se a consistência e robustez dos resultados devem adoptar-se, simultaneamente, vários métodos.

\subsubsection{Cálculo do índice PTF de Malmquist}

Neste trabalho, apresentamos dois métodos alternativos de cálculo do índice PTF de Malmquist, baseados no modelo fronteira DEA ("Data Envelopment Analysis") ${ }^{10}$ e na análise fronteira estocástica (AFE). A diferença essencial entre os dois métodos reside no facto do primeiro recorrer a métodos não paramétricos para estimação das funções distância e a partir destas calcular o índice PTF e suas componentes alteração da eficiência técnica (AE) ou catching-up e alteração tecnológica (AT) ou inovação tecnológica, enquanto no segundo se usam métodos paramétricos para o cálculo dos índices de AE, AT e respectivo índice PTF. Aparte esta diferença, os dois métodos adoptam o conceito de função distância, têm em vista a mesma finalidade e devem chegar a resultados idênticos e coerentes.

\subsubsection{Funções distância}

O índice de Malmquist é definido através de funções distância. Caso seja necessário, estas aplicam-se facilmente a tecnologias de produção multifactor e multiproduto, sem necessidade de assunção do comportamento económico forte de maximização do lucro ou de minimização do custo. Adicionalmente, as funções distância podem ser calculadas tanto na perspectiva dos factores, como na dos produtos. A primeira indica a mínima contracção, proporcional, possível de ocorrer no consumo de factores, dado o vector de produtos. A segunda representa a máxima expansão, proporcional, possível no vector de produtos, dado o consumo de factores. Neste trabalho, seleccionámos a 
Medição da evolução da produtividade total dos factores: o índice de Malmquist

orientação produto, por nos parecer que na tomada de decisão os agricultores tentam maximizar o nível de produção ${ }^{11}$, dado o conjunto de factores e não o inverso.

\subsection{Conjunto de possibilidades de produção e funções distância}

Para a definição da função distância, assumimos que as possibilidades tecnológicas das empresas sob observação podem ser descritas pelo conjunto $\mathrm{Y}$ de pares de combinações possíveis de factores e produtos (x,y). Mais especificamente, que a tecnologia de produção pode ser descrita pelo conjunto de possibilidades de produção, $\mathrm{P}(\mathrm{x})$, com:

(3) $\quad P(x)=\{y:(x, y)$ ? Y, i.e. $x$ permite produzir $y\}$

Assume-se, ainda, que a tecnologia satisfaz os seguintes axiomas (Coelli et al., 1998: cap. 3):

i) 0 ? P(x); para dado conjunto de factores é possível uma produção nula

(i.e. a inacção é possível);

ii) Uma combinação de produtos não nula requer uma de factores não nula;

iii) $\mathrm{P}(\mathrm{x})$ satisfaz a hipótese de forte dispensabilidade nos produtos; se y ? $\mathrm{P}(\mathrm{x})$ e $\mathrm{y}^{*}$ ? $\mathrm{y}$, então $\mathrm{y}^{*}$ ? $\mathrm{P}(\mathrm{x})^{12}$;

iv) $\mathrm{P}(\mathrm{x})$ satisfaz a hipótese de forte dispensabilidade de factores ; i. é. se $\mathrm{y}$ pode ser produzido com $\mathrm{x}$, então y pode ser produzido a partir de qualquer $\mathrm{x}^{*}$ ? $\mathrm{x}^{13}$;

v) $\mathrm{P}(\mathrm{x})$ é delimitado, fechado e convexo.

Pressupondo os axiomas anteriores, a função distância produto, $\mathrm{d}_{\mathrm{o}}(\mathrm{x}, \mathrm{y})$, é definida por:

$$
\mathrm{d}_{\mathrm{O}}(\mathrm{x}, \mathrm{y})=\min \{?:(\mathrm{y} / ?) ? \mathrm{P}(\mathrm{x})\}
$$

Admitindo que y é um elemento do conjunto de possibilidades de produção, $\mathrm{P}(\mathrm{x})$, o escalar (?) indicativo da função distância tomará valor igual ou inferior à unidade se y se localizar sobre a fronteira de possibilidades de produção (FPP) ou dentro do conjunto de possibilidades de produção, respectivamente. Como veremos em 2.2.2.2., conhecidos os valores das funções distância facilmente se calcula o índice PTF de Malmquist. 


\subsection{Representação gráfica da função distância}

A figura 1 ilustra o conceito de função distância produto, para um situação simples em que são produzidos dois produtos $\left(\mathrm{y}_{1}\right.$ e $\left.\mathrm{y}_{2}\right)$ utilizando o vector de factores $\mathrm{x}$. Os pontos $\mathrm{C}$ e $\mathrm{B}$ representam situações localizadas sobre a FPP, com ?=1, enquanto para o ponto A o escalar que quantifica a função distância é dado por $?=\mathrm{OA} / \mathrm{OB}<1$.

Figura 1

FUNÇÃO DISTÂNCIA PRODUTO E CONJUNTO DE POSSIBILIDADES DE PRODUÇÃO

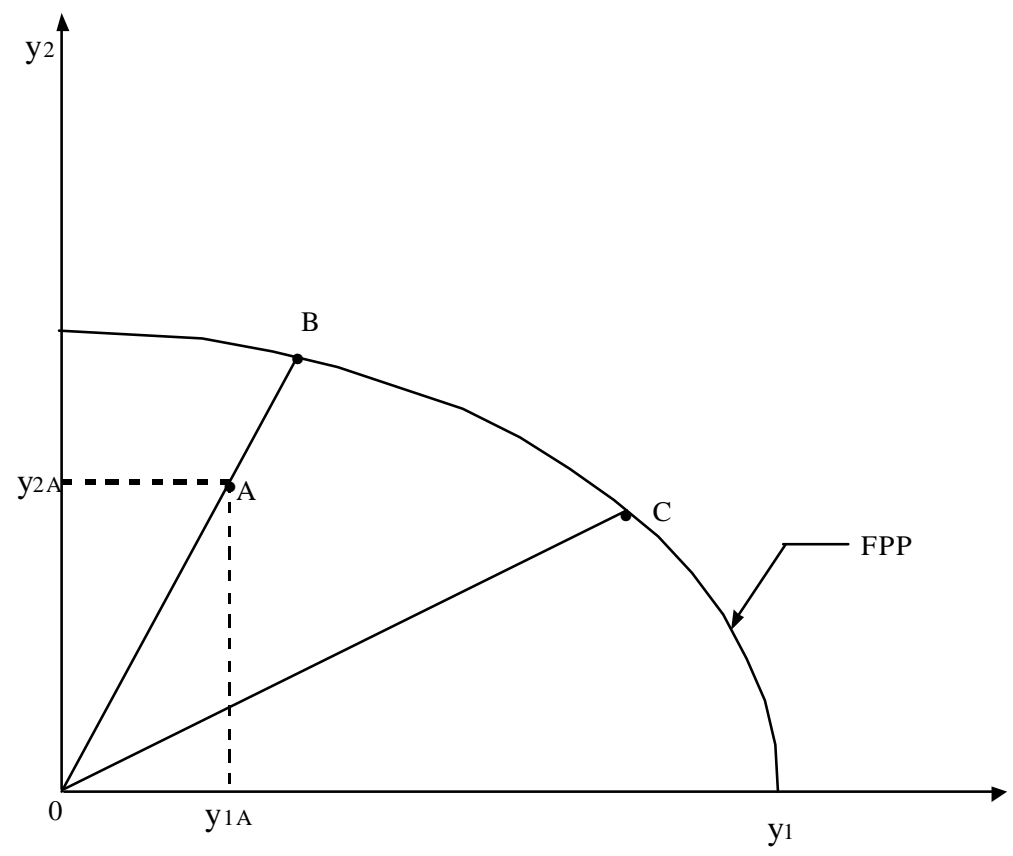




\subsubsection{Fórmula do índice PTF de Malmquist}

O índice de Malmquist permite quantificar a alteração da PTF entre dois pares de combinações de factores e produtos $(\mathrm{x}, \mathrm{y})$, através do cálculo do rácio das distâncias de cada par relativamente a uma fronteira comum. Seguindo Grifell-Tatjé e Lovell (1996), para certa unidade, o índice (produto orientado) PTF de Malmquist, entre os períodos s (período de base) e $t^{14}$ é dado por:

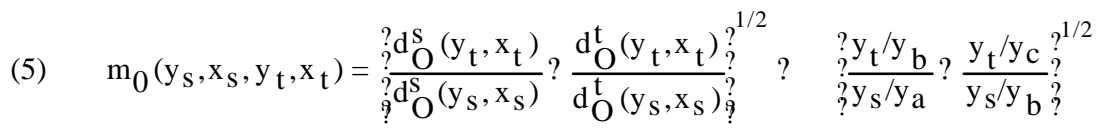

sendo $\mathrm{m}_{\mathrm{b}}\left(\mathrm{y}_{\mathrm{S}}, \mathrm{x}_{\mathrm{S}}, \mathrm{y}_{\mathrm{t}}, \mathrm{x}_{\mathrm{t}}\right)$ a média geométrica de dois índices de Malmquist ${ }^{15}$ (rácios entre funções distância), adoptando o primeiro como tecnologia referência a do período $\mathrm{s}$ e o segundo a do período $\mathrm{t}^{16}$. Em qualquer dos casos um valor do índice de Malmquist superior, igual ou inferior à unidade indica a ocorrência de crescimento, estagnação ou declínio da PTF.

Uma característica importante do índice PTF de Malmquist, salientada pela primeira vez por Färe et al. (1995), é que a expressão (5) é facilmente decomposta num produto entre o índice de alteração da eficiência técnica e o índice de alteração tecnológica, conforme (6).

$$
\mathrm{m}_{\mathrm{O}}\left(\mathrm{y}_{\mathrm{s}}, \mathrm{x}_{\mathrm{s}}, \mathrm{y}_{\mathrm{t}}, \mathrm{x}_{\mathrm{t}}\right) ? \frac{\mathrm{d}_{\mathrm{O}}^{\mathrm{t}}\left(\mathrm{y}_{\mathrm{t}}, \mathrm{x}_{\mathrm{t}}\right)}{\mathrm{d}_{\mathrm{O}}^{\mathrm{s}}\left(\mathrm{y}_{\mathrm{s}}, \mathrm{x}_{\mathrm{s}}\right)} \stackrel{?}{?} \frac{\mathrm{d}_{\mathrm{O}}^{\mathrm{s}}\left(\mathrm{y}_{\mathrm{t}}, \mathrm{x}_{\mathrm{t}}\right)}{\mathrm{d}_{\mathrm{O}}^{\mathrm{t}}\left(\mathrm{y}_{\mathrm{t}}, \mathrm{x}_{\mathrm{t}}\right)} ? \frac{\mathrm{d}_{\mathrm{O}}^{\mathrm{s}}\left(\mathrm{y}_{\mathrm{s}}, \mathrm{x}_{\mathrm{s}}\right)}{\mathrm{d}_{\mathrm{O}}^{\mathrm{t}}\left(\mathrm{y}_{\mathrm{s}}, \mathrm{x}_{\mathrm{s}}\right)} \stackrel{?}{?} \stackrel{?}{?}
$$

Em (6), a primeira componente (parte do lado direito da expressão fora do parênteses recto) indic a a alteração ocorrida nos níveis de eficiência técnica (AE) de Farrel (1957), entre os períodos t e s, indicando se o produtor se está a aproximar ou a afastar da melhor prática tecnológica. Conforme AE seja maior, igual ou inferior à unidade verifica-se uma melhoria, estagnação ou declínio, respectivamente, da performance relativa do produtor em relação à tecnologia disponível. A segunda componente (expressão dentro do parênteses recto) indica a alteração tecnológica (AT) auferida pelo produtor. Está a melhorar, estagnar ou regredir, em relação à melhor prática tecnológica, conforme AT seja superior, igual ou inferior à unidade, respectivamente. 
A figura 2 contempla a decomposição da PTF em AE e AT, assumindo-se a situação simples de uma tecnologia de rendimentos constantes à escala envolvendo a produção de um produto com um único factor, localizando-se a empresa nos pontos D e E para os períodos s e t, respectivamente. Em cada um dos períodos, a empresa está a produzir abaixo da fronteira de "melhor prática", havendo uma situação de ineficiência técnica. Utilizando as componentes da expressão (6) obtém-se:

$$
\begin{aligned}
& \mathrm{AE}=\frac{\mathrm{d}_{\mathrm{O}}^{\mathrm{t}}\left(\mathrm{y}_{\mathrm{t}}, \mathrm{x}_{\mathrm{t}}\right)}{\mathrm{d}_{\mathrm{O}}^{\mathrm{s}}\left(\mathrm{y}_{\mathrm{s}}, \mathrm{x}_{\mathrm{s}}\right)} ? \frac{\mathrm{y}_{\mathrm{t}} / \mathrm{y}_{\mathrm{c}}}{\mathrm{y}_{\mathrm{s}} / \mathrm{y}_{\mathrm{a}}}
\end{aligned}
$$

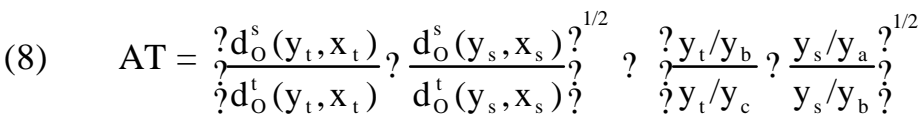

Os índices PTF de Malmquist e suas componentes AE e AT são índices locais, no sentido de que os seus valores podem variar entre produtores e no tempo. Em determinado momento, pode haver, simultaneamente, produtores que exibem padrões de eficiência técnica crescente e outros decrescente. De modo semelhante pode haver produtores que ao longo do tempo exibem um padrão de progresso técnico e outros de decadência técnica. Estas características dos índices de Malmquist permitem considerável flexibilidade na explicação do padrão de alteração da PTF entre produtores e ao longo do tempo.

A expressão (6) indica que, em aplicações empíricas, é necessário calcular quatro medidas de distância, o que pode ser feito recorrendo a técnicas de programação matemática ou econométricas. Estes métodos serão discutidos na secção seguinte. 
Figura 2

DECOMPOSIÇÃO DO ÍNDICE PTF DE MALMQUIST

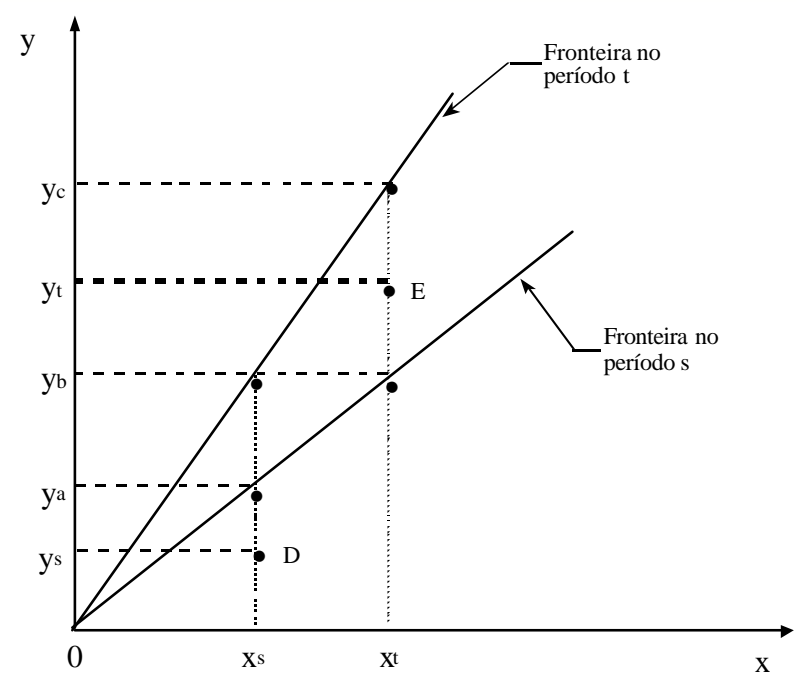

Uma questão adicional na quantificação empírica da PTF que deve ser enfatizada é a importância da propriedade adoptada quanto aos rendimentos de escala ${ }^{17}$ subjacentes à tecnologia de produção. Grifell-Tatjé e Lovell (1995), usando o exemplo simples uniproduto unifactor, demonstram que o índice PTF de Malmquist origina um enviesamento na evolução da produtividade total dos factores quando é assumida uma tecnologia de rendimentos variáveis à escala. Perante um acréscimo no consumo de factores verifica-se uma sobre ou subavaliação da PTF conforme se esteja na presença de rendimentos decrescentes ou crescentes à escala, respectivamente, sendo a direcção do enviesamento invertida se ocorrer uma contracção no consumo de factores. Neste contexto, apesar de constituir uma limitação, é indispensável que na estimação dos modelos que suportam o cálculo das funções distância supra referidas seja imposta a hipótese de ocorrência de rendimentos constantes à escala.

\subsubsection{Métodos de estimação das funções distância}

Os métodos frequentemente utilizados na estimação das funções distância inerentes ao índice PTF de Malmquist são o método de programação linear 
DEA sugerido por Färe et al. (1994) e o modelo AFE inicialmente sugerido por Aigner et al. (1977) e Meeusen e van den Broeck (1977).

\subsubsection{Método Data Envelopment Analysis}

Perante a disponibilidade de panel data e resolvendo diferentes problemas de programação linear DEA (Färe et al.,1994), para a empresa i e períodos s e t, podemos quantificar as quatro funções distância necessárias ao cálculo do índice PTF de Malmquist e suas componente AE e AT, conforme expressão (6). Assumindo uma tecnologia de rendimentos constantes à escala $(\mathrm{RCE})^{18}$, o cômputo das funções distância exige a resolução dos seguintes quatro problemas de programação linear $(\mathrm{PL})^{19}$ :

(9)

$$
\begin{gathered}
{\left[\mathrm{d}_{\mathrm{O}}^{\mathrm{t}}\left(\mathrm{y}_{\mathrm{t}}, \mathrm{x}_{\mathrm{t}}\right)\right]^{-1}=\max ?,} \\
\text { s.a: } \quad-? \mathrm{y}_{\mathrm{it}}+\mathrm{Y}_{\mathrm{t}} ? ? 0, \\
\quad \mathrm{x}_{\mathrm{it}}-\mathrm{X}_{\mathrm{t}} ? ? 0,
\end{gathered}
$$

$$
\begin{gathered}
{\left[\mathrm{d}_{\mathrm{O}}{ }^{\mathrm{s}}\left(\mathrm{y}_{\mathrm{s}}, \mathrm{x}_{\mathrm{s}}\right)\right]^{-1}=\max ?,} \\
\text { s.a: } \quad-? \mathrm{y}_{\mathrm{is}}+\mathrm{Y}_{\mathrm{s}} ? ? 0, \\
\mathrm{x}_{\mathrm{is}}-\mathrm{X}_{\mathrm{s}} ? ? 0,
\end{gathered}
$$

$$
\begin{aligned}
& {\left[\mathrm{d}_{\mathrm{O}}^{\mathrm{t}}\left(\mathrm{y}_{\mathrm{s}}, \mathrm{x}_{\mathrm{s}}\right)\right]^{-1}=\max ?,} \\
& \text { s.a: } \quad-? \mathrm{y}_{\mathrm{is}}+\mathrm{Y}_{\mathrm{t}} ? ? 0, \\
& \mathrm{x}_{\mathrm{is}}-\mathrm{X}_{\mathrm{t}} ? ? 0, \\
& ? ? 0,
\end{aligned}
$$

$$
\begin{aligned}
& {\left[\mathrm{d}_{\mathrm{O}}^{\mathrm{s}}\left(\mathrm{y}_{\mathrm{t}}, \mathrm{x}_{\mathrm{t}}\right)\right]^{-1}=\max ?,} \\
& \text { sa: } \quad-? \mathrm{y}_{\mathrm{it}}+\mathrm{Y}_{\mathrm{s}} ? ? 0, \\
& \mathrm{x}_{\mathrm{it}}-\mathrm{X}_{\mathrm{s}} ? ? 0, \\
& \quad ? ? 0 .
\end{aligned}
$$

Sendo :

?? $y_{\text {it }}$ e $\mathrm{y}_{\text {is }}$ os vectores, Mx1, das quantidades de produtos da empresa i nos períodos $\mathrm{t}$ e $\mathrm{s}$, respectivamente;

?? $\mathrm{x}_{\text {it }}$ e $\mathrm{x}_{\text {is }}$ os vectores, $\mathrm{Kx} 1$, das quantidades de factores da empresa i nos períodos $t$ e $s$, respectivamente; 
Medição da evolução da produtividade total dos factores: o índice

de Malmquist

?? $\mathrm{Y}_{\mathrm{t}} \mathrm{e} \mathrm{Y}_{\mathrm{S}}$ os vectores, $\mathrm{MxN}$, das quantidades de produtos para todas as $\mathrm{N}$ empresas nos períodos $\mathrm{te} \mathrm{s}$, respectivamente;

?? $\mathrm{X}_{\mathrm{t}}$ e $\mathrm{X}_{\mathrm{S}}$ os vectores, $\mathrm{KxN}$, das quantidades de factores para todas as $\mathrm{N}$ empresas nos períodos $\mathrm{te} \mathrm{s}$, respectivamente;

??? o vector de parâmetros, $\mathrm{Nx} 1$, das ponderações que permitem formar combinações lineares de produtos e factores, podendo assumir valores diferentes em cada um dos problemas;

??? é um escalar, inverso da função distância, com valor específico em cada um dos problemas.

Saliente-se que nos problemas de PL (11) e (12), cujas combinações produtos-factores são comparadas para tecnologias de diferentes períodos, $\mathrm{o}$ parâmetro ? não necessita obrigatoriamente de ser igual ou superior a um, exigência que se verifica no cálculo dos índices produto orientado de Farrel (1957) e reflectida, no nosso caso, no parâmetro ? de (4), com ? =1/?. Além disso, para cada empresa da amostra têm de resolver-se os quatro problemas de PL, implicando que para $\mathrm{N}$ empresas e $\mathrm{T}$ períodos se efectuarmos, sucessivamente, comparações entre períodos adjacentes têm de resolver-se $\mathrm{Nx}(3 \mathrm{~T}-2)$. Por exemplo, para $\mathrm{N}=26 \mathrm{e} \mathrm{T}=2$ têm de resolver-se 104 problemas de PL.

\subsection{Método da análise fronteira estocástica}

As medidas distância requeridas pelo índice PTF de Malmquist também podem ser extraídas a partir de uma "tecnologia paramétrica", representada por uma função fronteira produção ${ }^{20}$, custo ou lucro. No nosso caso, vamos ilustrar a aplicação desta metodologia considerando a seguinte função de produção fronteira estocástica

$$
\operatorname{lny}_{i t}=f\left(x_{i t}, t, ?\right)+v_{i t}-u_{i t} ; \quad i=1,2, \ldots, N ; t=1,2, \ldots, T ;
$$

em que: $\operatorname{lny}_{\text {it }}$ é o logaritmo natural da produção da iésima empresa no período $\mathrm{t}$; $\mathrm{f}($.) é a forma funcional adequada ao caso em estudo (e.g. translog); x é o vector (1xK) de factores, expressos também sob a forma de logaritmos; t é a trend temporal representativa da alteração tecnológica; ? é o vector dos parâmetros a serem estimados e associados ao vector de factores $\mathrm{x}$ e à variável $t ; v_{i t}$ são os erros aleatórios tradicionais, assumidos como i.i.d. e com distribuição normal $\mathrm{N}\left(0, ? \mathrm{v}^{2}\right)$ e independentes dos $\mathrm{u}_{\mathrm{it}}$; $\mathrm{u}_{\mathrm{it}}$ são considerados truncamentos (em zero) não negativos i.i.d. da variável 
aleatória de distribuição normal $\mathrm{N}\left(0, ? \mu^{2}\right)$, reflectindo os efeitos ineficiência técnica.

O conjunto de parâmetros da função (13) são estimados pelo método de máxima verosimilhança ${ }^{21}$. A eficiência técnica de cada empresa em cada ano pode ser calculada através da esperança condicional de $\exp \left(-\mathrm{u}_{\mathrm{it}}\right)$, dado o valor de $\mathrm{e}_{\mathrm{it}}=\mathrm{v}_{\mathrm{it}}-\mathrm{u}_{\mathrm{it}}$, utilizando a seguinte equação (Battese e Coelli, 1988) ${ }^{22}$ :

$$
\text { E? } \exp ?-\mathrm{u}_{\mathrm{it}} ? \mid \mathrm{e}_{\mathrm{it}} ? ? \frac{1-\mathrm{F}\left(\mathrm{s}_{\mathrm{A}} ? ? \mathrm{e}_{\mathrm{it}} / \mathrm{s}_{\mathrm{A}}\right)}{1 ? \mathrm{~F} ? \mathrm{e}_{\mathrm{it}} / \mathrm{s}_{\mathrm{A}} ?} \exp \left(? \mathrm{x}_{\mathrm{it}} ? \mathrm{~s}_{\mathrm{A}}^{2} / 2\right)
$$

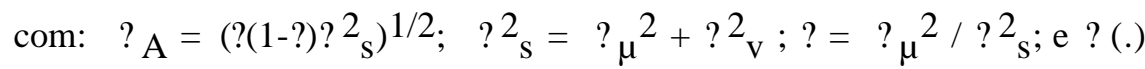
a função distribuição da variável aleatória normal reduzida.

Dada a distribuição de u it (variável aleatória não negativa), os índices de eficiência técnica, $\mathrm{ET}_{\text {it }}=\exp \left(-\mathrm{u}_{\mathrm{it}}\right.$ ? $\left._{\mathrm{it}}\right)$, variam entre zero e um, indicando este último plena eficiência técnica.

Estimada a função (13) e conhecidos os índices de eficiência técnica (ET), é possível calcular o índice PTF de Malmquist dado pela equação (6). Primeiro, entre os períodos t e s, AE é dada por:

$$
\mathrm{AE}=\mathrm{ET}_{\mathrm{it}} / \mathrm{ET}_{\mathrm{is}},
$$

com: $\mathrm{ET}_{\text {it }}=\mathrm{d}_{\mathrm{o}} \mathrm{t}\left(\mathrm{y}_{\mathrm{it}}, \mathrm{x}_{\mathrm{it}}\right)$ e $\mathrm{ET}_{\text {is }}=\mathrm{d}_{\mathrm{o}} \mathrm{s}\left(\mathrm{y}_{\mathrm{is}}, \mathrm{x}_{\mathrm{is}}\right)$.

Segundo, o índice de alteração tecnológica (AT), entre os dois períodos $t$ e s, pode ser calculado directamente a partir dos parâmetros estimados, recorrendo-se simplesmente ao valor derivada parcial da função de produção em relação à trend temporal nos pontos específicos s e t. Pressupondo que a alteração tecnológica é não neutra, variando AT com os níveis de utilização de factores, é sugerida a utilização da média geométrica entre os períodos $\mathrm{s}$ e t (Coelli et al., 1998), isto é:

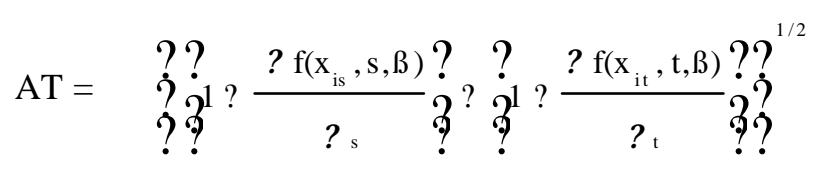

Multiplicando os valores obtidos em (15) e (16) obtém-se o índice PTF de Malmquist. Note-se que este índice, tal como no DEA, é perfeitamente possível de ser calculado directamente a partir das quatro medidas distância referidas em $(6)^{23}$, isto é, pelos rácios entre as quantidades produzidas e as 
previstas para a fronteiras de produção correspondentes aos vectores de factores nos períodos s e t.

Alternativamente ao método anterior, caso o número de observações o permita (i.e., estatisticamente, haja graus de liberdade suficientes), a medição da AT pode ser feita a partir da comparação de mudanças periódicas das funções produção seccionais estimadas. Neste caso, assume-se que as mudanças tecnológicas afectam todos os coeficientes da regressão, sendo estes utilizados como a "tecnologia de referência" no cálculo da produção, para a empresa i, inerente ao consumo de factores nos períodos t e s. Ainda que incidindo sobre a função custo dual, este método foi aplicado por Rebelo e Mendes (1997) ao sector bancário português.

\section{APLICAÇÃO DO ÍNDICE PTF DE MALMQUIST A EXPLORAÇÕES AGRÍCOLAS DO PLANALTO MIRANDÊS}

\subsection{Dados}

O objectivo desta parte do trabalho é elucidar a aplicação do índice de Malmquist à análise da produtividade dos factores (AE, AT e PTF), tendo por base os métodos ño paramétrico (DEA) e paramétrico (AFE baseada numa função de produção) discutidos em pontos anteriores. Assim sendo, para cálculo dos indicadores de produtividade, assumimos a empresa agrícola como uma organização que transforma, anualmente, um produto $(\mathrm{y}=$ produto bruto, igual à soma dos produto bruto vegetal com o animal, em contos), a partir do consumo de quatro factores produtivos: $\mathrm{S}=$ superfície agrícola útil cultivada (SAU/ha); $\mathrm{L}=$ trabalho (UTA); $\mathrm{K}=$ capital (amortizações e reintegrações e despesas com reparação e conservação de edifícios e equipamentos, em contos); $\mathrm{F}=$ outros factores (sementes, fertilizantes, combustíveis e lubrificantes e outros encargos variáveis, em contos).

Os dados de base foram recolhidos a partir de informação contabilístic a de explorações agrícolas localizadas na Zona Homogénea do Planalto Mirandês e aderentes ao sistema de Rede de Informação de Contabilidade Agrícola (RICA) implementado pelo Ministério da Agricultura, Desenvolvimento Rural e Pescas (MADRP) ${ }^{24}$. Os dados referem-se aos anos decorrentes entre 1990 e 1997, tendo-se excluído da amostra as explorações que para algumas variáveis apresentavam registos com valores não positivos. Por outro lado, conjuntamente com esta triagem, apenas se consideraram as explorações que foram observadas ao longo de todo o período, de modo a 
construir-se um panel equilibrado ${ }^{25}$ constituído por 26 empresas agrícolas observadas durante 8 anos consecutivos ${ }^{26}$. As variáveis quantificadas em valores monetários foram expressas a preços do ano base (1990), usando-se o índice de preços dos produtos agrícolas para o produto bruto e o deflactor do produto interno bruto para o capital e outros factores.

O quadro 1 inclui informação estatística sobre as variáveis utilizadas, assim como sobre a evolução da produtividade aparente ou parcial (produto bruto/consumo) dos factores produtivos. Salienta-se que no período em análise: cerca de $78 \%$ do produto bruto total é de origem animal, aumentando esta proporção de 67,1\% em 1990 para 81,3\% em 1997, o que indicia uma mudança no padrão de especialização produtiva, com reforço das actividades pecuárias, em detrimento das vegetais, nomeadamente dos cereais; da SAU cultivada apenas $6 \%$ é de regadio; do trabalho utilizado $94 \%$ é de origem familiar.

Tomando como referência a média, verifica-se que, entre 1997 e 1990 (gráfico 1), o produto bruto teve uma evolução acumulada de $+7,1 \%$, enquanto nos factores produtivos as variações acumuladas são de $-3,9 \%$, $26,9 \%,-8,1 \%$ e $+74,9 \%$, para a SAU, trabalho, outros factores e capital, respectivamente. Da evolução conjugada do produto e do consumo de factores resultou um comportamento anual, relativamente, homogéneo dos quatro índices de produtividade aparente (gráfico 2), ocorrendo a pior e melhor situação em 1994 e 1996. No primeiro caso, devido ao mau ano agrícola de 1994, comparativamente ao bom ano de 1993, verificou-se um decréscimo de $46 \%$ da produção vegetal apenas parcialmente compensado pelo aumento de 6,6\% do produto bruto animal. Para os resultados de 1996 contribuíram, em relação ao ano normal de 1995 , os acréscimos de $26 \%$ e $32,6 \%$ no produto bruto animal e vegetal, respectivamente.

Em termos acumulados ${ }^{27}$ (gráfico 3), verificou-se crescimento da produtividade aparente da SAU $(+11,4 \%)$, do trabalho $(+46,6 \%)$ e de outros factores $(+16,4 \%)$ e um decréscimo $(-38,8 \%)$ para o capital, indiciando que, no período em análise, ocorreu uma melhoria da produtividade total dos factores, a qual pode ter resultado tanto de um melhor aproveitamento dos recursos produtivos (maior eficiência técnica) ou de melhorias tecnológicas (deslocação da fronteira de produção), como veremos em 3.2.2. 
Medição da evolução da produtividade total dos factores: o índice de Malmquist

\section{Quadro 1}

INFORMAÇÃO ESTATÍSTICA SOBRE AS VARIÁVEIS DOS MODELOS

\begin{tabular}{|c|c|c|c|c|c|c|c|c|}
\hline & 1990 & 1991 & 1992 & 1993 & 1994 & 1995 & 1996 & 1997 \\
\hline \multicolumn{9}{|l|}{$\mathrm{y}=$ Produto bruto $*$} \\
\hline Máximo & 7986 & 8779 & 12417 & 16069 & 10217 & 11918 & 14771 & 14159 \\
\hline Mínimo & 617 & 722 & 432 & 564 & 736 & 257 & 491 & 522 \\
\hline Média & 3411 & 3415 & 3791 & 3978 & 3525 & 3587 & 4549 & 3652 \\
\hline Coef. Variação (\%) & 63,2 & 67,0 & 93,5 & 91,1 & 78,3 & 85,0 & 87,1 & 93,1 \\
\hline \multicolumn{9}{|l|}{$\mathrm{S}=\mathrm{SAU}$ (ha) } \\
\hline Máximo & 65 & 51,2 & 76,9 & 55,4 & 70,4 & 99,8 & 80 & 62 \\
\hline Mínimo & 10,2 & 10,7 & 12,2 & 11,5 & 10,8 & 10,7 & 10,6 & 10,2 \\
\hline Média & 31 & 28,2 & 31,5 & 31,1 & 28,1 & 32,2 & 30,1 & 29,8 \\
\hline Coef. Variação (\%) & 46,3 & 42,0 & 52,0 & 44,5 & 52,3 & 55,9 & 50,0 & 43,9 \\
\hline \multicolumn{9}{|l|}{$\mathrm{L}=$ Trabalho (UTA) } \\
\hline Máximo & 2,84 & 2,77 & 2,4 & 2,62 & 2,8 & 3,21 & 2,36 & 2,17 \\
\hline Mínimo & 0,95 & 0,71 & 0,73 & 0,72 & 0,67 & 0,63 & 0,57 & 0,3 \\
\hline Média & 1,93 & 1,65 & 1,64 & 1,76 & 1,73 & 1,47 & 1,19 & 1,41 \\
\hline Coef. Variação (\%) & 25,1 & 26,3 & 23,7 & 23,8 & 25,8 & 34,1 & 32,2 & 29,3 \\
\hline \multicolumn{9}{|l|}{$\mathrm{K}=$ Capital ${ }^{*}$} \\
\hline Máximo & 1060 & 952 & 971 & 899 & 1027 & 1040 & 1053 & 1030 \\
\hline Mínimo & 1 & 0,9 & 0,8 & 0,8 & 0,7 & 0,7 & 0,7 & 0,7 \\
\hline Média & 171 & 191 & 228 & 241 & 269 & 253 & 278 & 299 \\
\hline Coef. Variação (\%) & 123,2 & 121,5 & 99,5 & 95,0 & 97,5 & 100,3 & 101,1 & 90,9 \\
\hline \multicolumn{9}{|l|}{$\mathrm{F}=$ Outros factores $*$} \\
\hline Máximo & 5713 & 5261 & 4930 & 4131 & 5280 & 4775 & 6269 & 4902 \\
\hline Mínimo & 116 & 135 & 109 & 131 & 128 & 110 & 92 & 109 \\
\hline Média & 1266 & 1226 & 1172 & 1347 & 1275 & 1383 & 1492 & 1164 \\
\hline Coef. Variação (\%) & 97,5 & 96,3 & 97,2 & 93,5 & 114,8 & 100,2 & 106,8 & 111,8 \\
\hline \multicolumn{9}{|c|}{ Produtividade aparente } \\
\hline P.bruto/SAU* & 110 & 121 & 120 & 128 & 125 & 111 & 151 & 123 \\
\hline P.bruto/Trabalho* & 1767 & 2070 & 2312 & 2260 & 2038 & 2440 & 3823 & 2590 \\
\hline P.bruto/Capital & 19,95 & 17,88 & 16,63 & 16,51 & 13,10 & 14,18 & 16,36 & 12,21 \\
\hline P.bruto/O. factores & 2,69 & 2,79 & 3,23 & 2,95 & 2,76 & 2,59 & 3,05 & 3,14 \\
\hline
\end{tabular}

* Em contos, a preços constantes de 1990. 
Gráfico 1

EVOLUÇÃO ACUMULADA DO P. BRUTO E DO CONSUMO

DE FACTORES $(1990=1,000)$

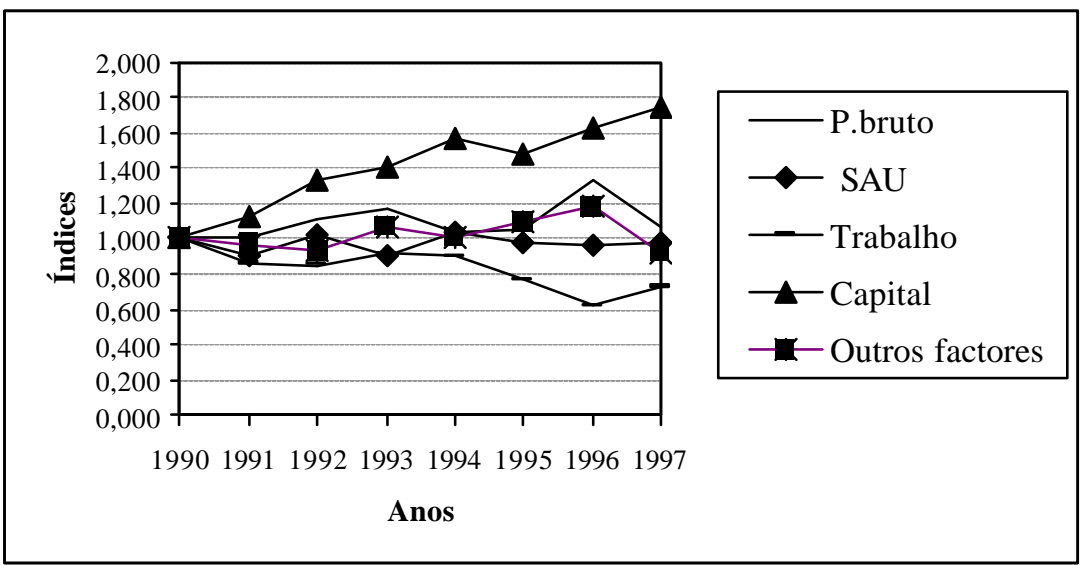

Gráfico 2

EVOLUÇÃO ANUAL DA PRODUTIVIDADE APARENTE

DOS FACTORES $(1990=1,000)$

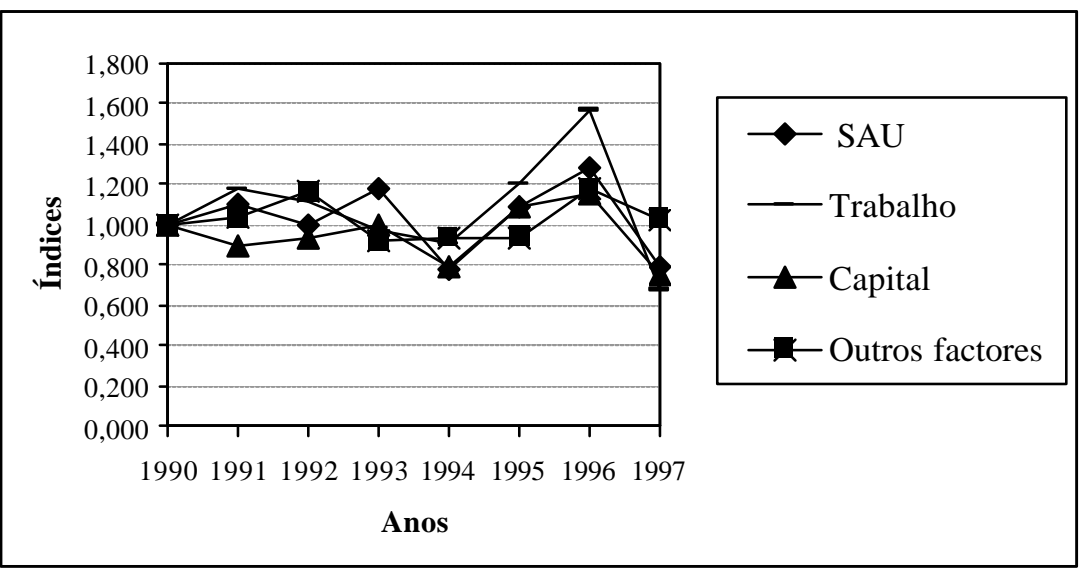




\section{Gráfico 3 \\ EVOLUÇÃO ACUMULADA DA PRODUTIVIDADE APARENTE DOS FACTORES $(1990=1,000)$}

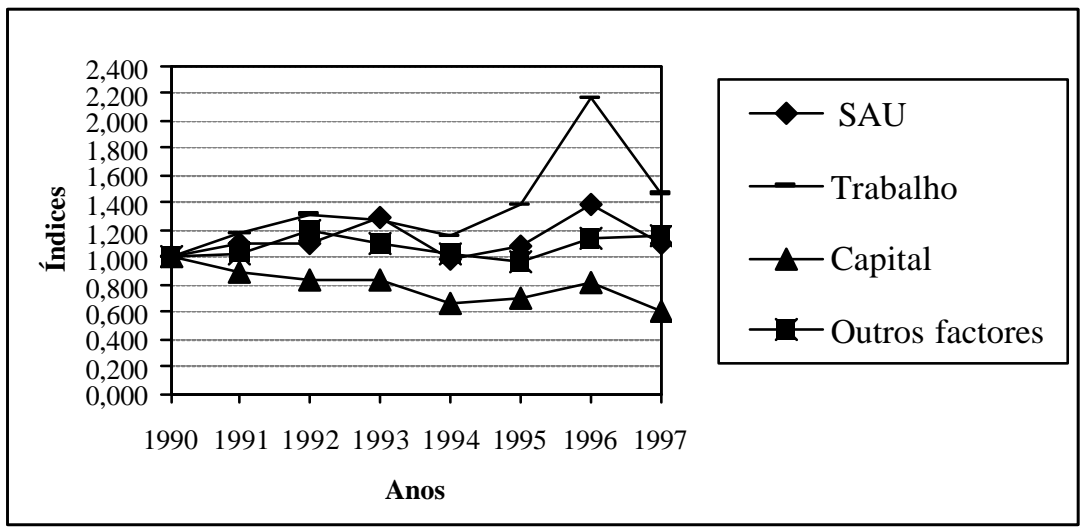

\subsection{Resultados}

\subsubsection{Modelos estimados}

As medidas das funções distância DEA expressas pelas equações (9) a (12) e concomitantes medidas da eficiência técnica (ET), do índice PTF de Malmquist e das suas componentes AE e AT, para anos adjacentes e cada uma das 26 explorações agrícolas (resolução de 572 problemas de PL), foram estimadas utilizando o programa DEAP (Coelli, 1996b).

Para aplicação do método AFE, isto é estimação das equações (13) e (14), assumimos que a tecnologia de produção da "agricultura" do Planalto Mirandês é susceptível de ser caracterizada por uma função de produção fronteira estocástica translogarítmica, sendo o produto bruto de cada exploração agrícola função de: quatro factores produtivos (terra, trabalho, capital e outros factores); da variável trend temporal t, a qual está especificada para uma situação de alteração tecnológica não neutra (interdependência com o consumo de factores); e do termo estocástico erro, tendo este duas componentes i.i.d., $v_{\text {it }}$ (erro estatístico tradicional de distribuição normal de média zero e variância homocedástica) e $\psi_{t}$ (erro estocástico, não negativo, com distribuição semi-normal de média zero e 
variância homocedástica). Nestas condições, assume-se a seguinte função de produção:

$$
\begin{aligned}
\ln \left(\mathrm{y}_{\mathrm{it}}\right)= & ?_{0}+?_{\mathrm{S}} \ln \left(\mathrm{S}_{\mathrm{it}}\right)+?_{\mathrm{L}} \ln \left(\mathrm{L}_{\mathrm{it}}\right)+?_{\mathrm{K}} \ln \left(\mathrm{K}_{\mathrm{it}}\right)+?_{\mathrm{F}} \ln \left(\mathrm{F}_{\mathrm{it}}\right)+1 / 2\left\{?_{\mathrm{SS}}\left[\ln \left(\mathrm{S}_{\mathrm{it}}\right)\right]^{2}\right. \\
& \left.+?_{\mathrm{LL}}\left[\ln \left(\mathrm{L}_{\mathrm{it}}\right)\right]^{2}+?_{\mathrm{KK}}\left[\ln \left(\mathrm{K}_{\mathrm{it}}\right)\right]^{2}+?_{\mathrm{FF}}\left[\ln \left(\mathrm{F}_{\mathrm{it}}\right)\right]^{2}\right\}+1 / 2\left[?_{\mathrm{SL}} \ln \left(\mathrm{S}_{\mathrm{it}}\right) \ln \left(\mathrm{L}_{\mathrm{it}}\right)\right. \\
& +?_{\mathrm{SK}} \ln \left(\mathrm{S}_{\mathrm{it}}\right) \ln \left(\mathrm{K}_{\mathrm{it}}\right)+?_{\mathrm{SF}} \ln \left(\mathrm{S}_{\mathrm{it}}\right) \ln \left(\mathrm{F}_{\mathrm{it}}\right)+?_{\mathrm{LS}} \ln \left(\mathrm{L}_{\mathrm{t}}\right) \ln \left(\mathrm{S}_{\mathrm{it}}\right)+?_{\mathrm{LK}} \ln \left(\mathrm{L}_{\mathrm{it}}\right) \ln \left(\mathrm{K}_{\mathrm{it}}\right) \\
& +?_{\mathrm{LF}} \ln \left(\mathrm{L}_{\mathrm{it}}\right) \ln \left(\mathrm{F}_{\mathrm{it}}\right)+?_{\mathrm{KS}} \ln \left(\mathrm{K}_{\mathrm{it}}\right) \ln \left(\mathrm{S}_{\mathrm{it}}\right)+?_{\mathrm{KL}} \ln \left(\mathrm{K}_{\mathrm{it}}\right) \ln \left(\mathrm{L}_{\mathrm{it}}\right)+?_{\mathrm{KF}} \ln \left(\mathrm{K}_{\mathrm{it}}\right) \ln \left(\mathrm{F}_{\mathrm{it}}\right) \\
& \left.+?_{\mathrm{FS}} \ln \left(\mathrm{F}_{\mathrm{it}}\right) \ln \left(\mathrm{S}_{\mathrm{it}}\right)+?_{\mathrm{FL}} \ln \left(\mathrm{F}_{\mathrm{it}}\right) \ln \left(\mathrm{L}_{\mathrm{it}}\right)+?_{\mathrm{FK}} \ln \left(\mathrm{F}_{\mathrm{it}}\right) \ln \left(\mathrm{K}_{\mathrm{it}}\right)\right]+?_{\mathrm{t}} \mathrm{t}+?_{\mathrm{tt}}\left(\mathrm{t}^{2} / 2\right) \\
& +?_{\mathrm{St}} \ln \left(\mathrm{S}_{\mathrm{it}}\right) \mathrm{t}+?_{\mathrm{Lt}} \ln \left(\mathrm{L}_{\mathrm{it}} \mathrm{t}\right)+?_{\mathrm{Kt}} \ln \left(\mathrm{K}_{\mathrm{it}}\right) \mathrm{t}+?_{\mathrm{Ft}} \ln \left(\mathrm{F}_{\mathrm{it}}\right) \mathrm{t}+\mathrm{v}_{\mathrm{it}}-\mathrm{u}_{\mathrm{it}} ; \\
& \mathrm{i}=1, \ldots, 26 ; \mathrm{t}=1, \ldots, 8 .
\end{aligned}
$$

Por outro lado, dado que o índice PTF de Malmquist deve ser calculado assumindo uma tecnologia de rendimentos constantes à escala (homogeneidade linear no consumo dos factores), à função (17) foram impostas as seguintes restrições:

$$
\begin{aligned}
& ?_{\mathrm{S}}+?_{\mathrm{L}}+?_{\mathrm{K}}+?_{\mathrm{F}}=1 ?_{\mathrm{SS}}+?_{\mathrm{SL}}+?_{\mathrm{SK}}+?_{\mathrm{SF}}=0 \quad ?_{\mathrm{LL}}+?_{\mathrm{LS}}+?_{\mathrm{LK}}+?_{\mathrm{LF}}=0 \\
& ?_{\mathrm{KK}}+?_{\mathrm{KS}}+?_{\mathrm{KL}}+?_{\mathrm{KF}}=0 \quad ?_{\mathrm{FF}}+?_{\mathrm{FS}}+?_{\mathrm{FL}}+?_{\mathrm{FK}}=0 ?_{\mathrm{St}}+?_{\mathrm{Lt}}+?_{\mathrm{Kt}}+?_{\mathrm{Ft}}=0
\end{aligned}
$$

A utilização de (18) permite a normalização do produto bruto e do consumo de factores em termos de um factor, dividindo-os a todos por este. No nosso caso, utilizámos, arbitrariamente ${ }^{28}$, o trabalho, passando tanto a produção como os restantes factores a ser expressos em unidades por UTA.

Por outro lado, previamente à estimação econométrica da função (17), além da imposição de (18) e da simetria nos parâmetros de segunda ordem $\left(?_{\mathrm{SL}}=?_{\mathrm{LS}} ; ?_{\mathrm{SK}}=?_{\mathrm{KS}} ; ?_{\mathrm{SF}}=?_{\mathrm{FS}} ; ?_{\mathrm{LK}}=?_{\mathrm{KL}} ; ?_{\mathrm{LF}}=?_{\mathrm{FL}} ; ?_{\mathrm{KF}}=?_{\mathrm{FK}}\right)$, os dados de cada variável foram escalonados à volta da respectiva média geométrica das 208 observações. Neste contexto, (17) deve ser interpretada como uma aproximação, na vizinhança desse ponto, da verdadeira função de produção ${ }^{29}$. Uma vez que o logaritmo natural de um é zero, esta forma de ordenação dos dados facilita o cálculo das elasticidades no ponto médio e a execução de testes locais.

Observados os procedimentos anteriores, os parâmetros do modelo definido pela função (17) foram estimados pelo método da máxima verosimilhança, sendo os índices individuais de eficiência técnica estimados aplicando (15), utilizando-se o software FRONTIER 4.1 Coelli (1996a). No anexo 1, quadro 1 , constam as estimativas dos parâmetros do modelo fronteira estocástico. 


\subsection{2. Índices de eficiência técnica, de alteração da eficiência técnica, de alteração tecnológica e da PTF: Métodos DEA e AFE}

No método DEA, os índices individuais de ET em cada ano são calculados através da resolução do problema de PL em que se assume como tecnologia de referência a do próprio ano, ou seja, adoptando a equação (9) ou (10). No método AFE, os índices de ET são estimados através da expressão (14). No quadro 2 apresentam-se as médias geométricas anuais dos índices calculados por ambos os métodos ${ }^{30}$.

Perante os resultados obtidos, ainda que com algumas diferenças, globalmente, os dois índices ${ }^{31}$ apontam para a mesma conclusão: se, no período em análise, todas as explorações agrícolas do Planalto Mirandês tivessem adoptado a melhor prátic a tecnológica, o produto bruto poderia, anualmente, ser superior em cerca de $33,9 \%$ ou 35,3\% (produto eficiente/produto observado, i.e. 1/ET) ao observado, conforme a eficiência técnica seja calculada pelas metodologias DEA ou AFE, respectivamente.

Quadro 2

ÍNDICES ANUAIS DE ET (MÉDIA GEOMÉTRICA)

\begin{tabular}{lcc}
\hline Ano & $\begin{array}{c}\text { Eficiência técnica } \\
\text { DEA }\end{array}$ & $\begin{array}{c}\text { Eficiência técnica } \\
\text { AFE }\end{array}$ \\
\hline 1990 & 0,765 & 0,729 \\
1991 & 0,792 & 0,763 \\
1992 & 0,613 & 0,734 \\
1993 & 0,747 & 0,756 \\
1994 & 0,767 & 0,758 \\
1995 & 0,810 & 0,682 \\
1996 & 0,823 & 0,785 \\
1997 & 0,687 & 0,711 \\
\hline Média global & 0,747 & 0,739 \\
\hline
\end{tabular}

$\mathrm{O}$ quadro 3 inclui as médias geométricas anuais dos índices AE, AT e de PTF referidos na equação (6) e calculados, para cada exploração e par de anos adjacentes, recorrendo no DEA aos valores do parâmetro ? assumidos em cada um dos problemas de PL (9) a (12) e no AFE a (15) e (16). 
Quadro 3

ÍNDICES DE AE, AT E PTF: MÉTODOS DEA E AFE

\begin{tabular}{lccc|ccc}
\hline & \multicolumn{3}{c|}{ DEA } & \multicolumn{3}{c}{ AFE } \\
\hline & AE & AT & PTF & AE & AT & PTF \\
$1991-90$ & 1,036 & 1,031 & 1,068 & 1,047 & 0,988 & 1,035 \\
$1992-91$ & 0,774 & 1,187 & 0,919 & 0,961 & 0,996 & 0,958 \\
$1993-92$ & 1,218 & 0,836 & 1,018 & 1,031 & 1,007 & 1,037 \\
$1994-93$ & 1,027 & 1,054 & 1,082 & 1,002 & 1,019 & 1,022 \\
$1995-94$ & 1,056 & 0,834 & 0,880 & 0,900 & 1,029 & 0,926 \\
$1996-95$ & 1,017 & 1,331 & 1,354 & 1,152 & 1,037 & 1,195 \\
$1997-96$ & 0,834 & 1,066 & 0,889 & 0,906 & 1,046 & 0,947 \\
\hline Acumulado 97-90 & 0,898 & 1,276 & 1,146 & 0,976 & 1,127 & 1,100 \\
\hline
\end{tabular}

Nos gráficos 4 e 5 estão representados os índices acumulados de AE, AT e PTF calculados pelos métodos DEA e AFE, respectivamente. No DEA, aqueles índices apresentam flutuações mais acentuadas que no AFE, resultado a que não é alheio o facto de ser um método determinista cujos desvios em relação à fronteira de produção, sejam de origem aleatória ou não, são considerados ineficiência técnica. Por outro lado, o gráfico 4 permite-nos ainda visualizar que no DEA, os índices AE e AT têm uma evolução oposta, estando negativamente correlacionados (para as 182 observações relativas ao conjunto de pares de anos adjacentes, o coeficiente de correlação de Pearson é de $-0,292$ e o de Spearman é de $-0,313$, ambos estatisticamente significativos a 5\%). Pelo contrário, no AFE pode ser rejeitada uma correlação linear entre os dois indicadores (os coeficientes de correlação linear de Pearson e Spearman são ambos estatisticamente não significativos), embora as médias geométricas anuais evoluam no mesmo sentido e quase de forma sobreposta (gráfico 5). 
Medição da evolução da produtividade total dos factores: o índice de Malmquist

Gráfico 4

ÍNDICES ACUMULADOS DE AE, AT E PTF - RESULTADOS DEA

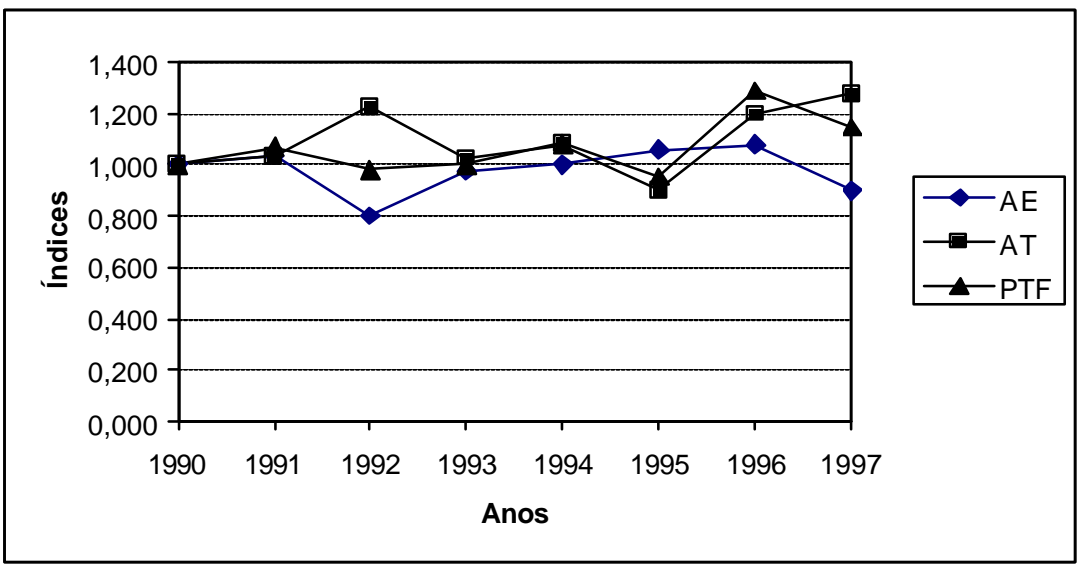

Gráfico 5

ÍNDICES ACUMULADOS DE AE, AT E PTF - RESULTADOS AFE

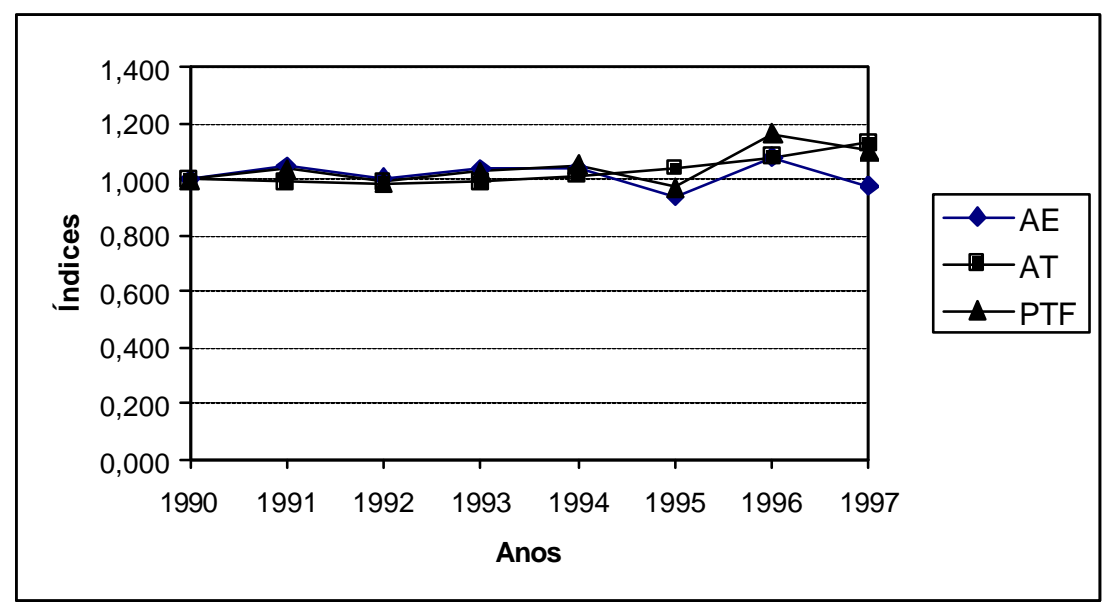

No gráfico 6 comparam-se os índices acumulados PTF de Malmquist calculados pelo DEA e pelo AFE, evoluindo os mesmos de forma similar e com valores muito semelhantes. Estatisticamente estão linearmente 
correlacionados de modo positivo, tendo os coeficientes de correlação de Pearson e Spearman valores de 0,899 e de 0,920, respectivamente (ambos estatisticamente significativos a $1 \%$ ).

\section{Gráfico 6}

ÍNDICES PTF ACUMULADOS - RESULTADOS DEA E AFE

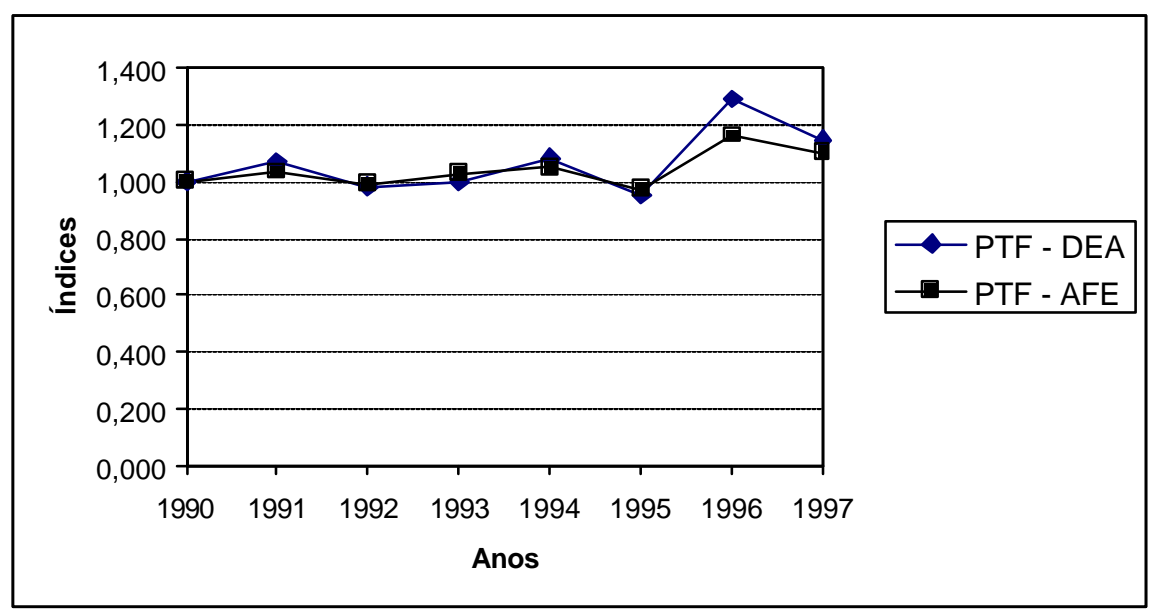

Perante os resultados observados, podemos assim inferir que entre 1997 e 1990 ocorreu uma variação positiva na produtividade total dos factores (o índice PTF de Malmquist teve uma variação acumulada de 14,6\% e 10,0\%, conforme seja medida pelo métodos DEA ou AFE, respectivamente), resultante do progresso tecnológico (27,6\% e 12,7\%, segundo o DEA ou o AFE, respectivamente), pois o efeito catching-up (alteração da eficiência técnica) foi ligeiramente negativo $(-10,2 \%$ pelo DEA e $-2,4 \%$ pelo AFE). Em síntese, eventualmente, devido a investimentos em novos equipamentos e novas tecnologias, as explorações agrícolas do Planalto Mirandês adoptaram estratégias indutoras de variações positivas na fronteira de produção (progresso tecnológico), mas a generalidade delas foi incapaz de se aproximar dessa fronteira (crescente ineficiência técnica). Por outro lado, estamos cientes de que os resultados, e respectivas conclusões retiradas sobre o comportamento das explorações agrícolas do Planalto Mirândes, podem ser refinados e melhorados se for possível aceder, além da contida na RICA, a informação adicional sobre variáveis caracterizadoras das explorações e dos agricultores incluídos na amostra (entre outras, características dos recursos 
físicos da exploração, idade do agricultor, formação profissional, recurso a serviços de extensão, capacidade de aceder ao mercado e à informação) e susceptíveis de influenciar os índices de eficiência técnica e de produtividade total dos factores.

\section{CONCLUSÕES}

As permanentes alterações ocorridas na envolvente económica e social resultantes do processo de globalização, do incremento da produção de bens invisíveis e das mutações tecnológicas tornam, cada vez mais, difícil e complexa a medição da performance da economia e das organizações produtivas.

Perante a crescente globalização dos mercados e liberalização das trocas, a competitividade do sistema económico é uma preocupação central de qualquer nação. Dependendo a competitividade de um país do crescimento sustentado da produtividade das organizações produtivas (com fins lucrativos ou não) que integram essa economia, é de grande importância conseguir-se um indicador capaz de captar as fontes e aferir de forma sintética, objectiva e adequada a evolução da produtividade. Esta medida é o índice de produtividade total dos factores, susceptível de ser quantificado através dos números índices de Malmquist, Fischer ou Törnqvist.

Neste estudo, prestamos especial atenção ao índice PTF de Malmquist, porque relativamente aos de Fischer e Törnqvist, apresenta as seguinte vantagens: para estimação da fronteira, via DEA ou AFE, não se requer informação sobre preços; não se necessita de assumir que todas as empresas são produtivamente eficientes; dispensa-se a assunção de comportamentos optimizadores, como sejam mínimo custo ou máximo rendimento; permite-se a decomposição do índice PTF em índices de alteração da eficiência técnica e da alteração tecnológica, ao longo do tempo. A principal fraqueza do índice de Malmquist reside no facto de apenas ser aplicável a situações de panel data, enquanto os Fischer e Törnqvist podem ser aplicados apenas a dois pontos de dados.

O índice PTF de Malmquist é calculado a partir de funções distância, recorrendo a métodos deterministas não paramétricos (DEA) ou a funções estocásticas paramétricas (AFE), havendo programas informáticos disponíveis (DEAP e FRONTIER 4.1, respectivamente) que, apesar da complexidade analítica envolvida, permitem facilmente a sua estimação.

Para ilustrarmos o processo de cálculo do índice PTF de Malmquist, aplicaram-se os métodos DEA e AFE a um panel equilibrado de 26 
explorações agrícolas para o período 1990-97. Genericamente, os resultados obtidos por ambas as metodologias são consistentes e permitem ver que: (1) caso todas as explorações agrícolas observadas adoptassem a melhor prática tecnológica, i.e., fossem tecnicamente eficientes, o produto agrícola bruto anual poderia, em média, ser superior em cerca de 34\% (DEA) ou 35\% (AFE) relativamente ao observado; (2) no período em análise, apesar de algumas flutuações anuais, ocorreu uma evolução positiva da PTF, 14,6\% e $10 \%$ segundo o DEA e o AFE, respectivamente; (3) o comportamento da PTF resultou do progresso ou inovação tecnológica observada (AT igual $27,6 \%$ no DEA e a $12,7 \%$ no AFE), cujo efeito foi, no entanto, amortecido pela evolução negativa da eficiência técnica $(-10,2 \%$ no DEA e $-2,4 \%$ no AFE); (4) no Planalto Mirandês foi possível observar que algumas explorações agrícolas adoptaram estratégias indutoras de variações positivas na fronteira de produção (progresso tecnológico), mas a generalidade delas foi incapaz de se aproximar dessa fronteira (crescente ineficiência técnica).

Em síntese, os métodos fronteira e os números índices, em especial o de Malmquist, constituem "bons" e flexíveis instrumentos de análise da eficiência produtiva e da produtividade total dos factores. No entanto, não podíamos deixar de referir algumas condicionantes a ter em mente quando se estão a modelizar e analisar casos concretos, de modo a evitarem-se resultados falaciosos. Referimos, nomeadamente:

?? O tratamento de factores produtivos e/ou produtos como bens homogéneos quando na realidade são heterogéneos e a exclusão de um importante factor ou produto conduzem a resultados enviesados.

?? Como os índices de eficiência produtiva (ou valores da função distância) são apenas relativos às melhores empresas da amostra, a existência de "outliers" influencia fortemente a forma e a localização da fronteira produtiva e, por consequência, os resultados finais.

?? Quando se detecta que uma determinada empresa apresenta um baixo e/ou instável índice de PTF, comparativamente a empresas congéneres da indústria, este resultado pode dever-se, por exemplo, tanto à incapacidade dos gestores introduzirem inovações tecnológicas e/ou utilizarem adequadamente os recursos disponíveis, como à adopção da escala produtiva inadequada, a diferenças qualitativas nos processos produtivos, factores e produtos ou simplesmente a erros de especificação na modelização no comportamento da empresa (erros de medição das variáveis e inclusão ou exclusão de variáveis relevantes).

?? Na comparação de índices de eficiência de amostras diferentes tem de ter-se em mente que cada um deles apenas reflecte o que se passa no interior da própria amostra, nada informando acerca do que se passa numa outra amostra. 
Por tudo isto se pode concluir que, na análise da produtividade total dos factores de casos concretos, os resultados finais devem ser sempre perspectivados e interpretados à luz das técnicas e metodologias utilizadas e que a adopção de vários métodos de estimação é um importante veículo de reforço da robustez dos resultados e das conclusões a reter.

\section{NOTAS}

* Texto da Lição Síntese apresentada nas Provas de Agregação, área científica de Economia - Teoria Económica e Métodos Quantitativos, na Universidade de Trás-osMontes e Alto Douro, em 19 de Setembro de 2000.

${ }^{1}$ Naturalmente que, para o cumprimento eficaz da missão das empresas, contribuem factores como o enquadramento macroeconómico, o funcionamento dos mercados, os sistemas educacional, jurídico e fiscal e as infra-estruturas físicas e tecnológicas.

${ }^{2}$ Medida de produtividade que envolve todos os factores de produção. Medidas de produtividade, como produtividade do trabalho, do capital, da terra ou de qualquer outro factor são conhecidas por medidas parciais da produtividade. Em qualquer dos casos, a medição das alterações da produtividade envolve necessariamente a quantificação de alterações nos níveis de produção assim como na concomitante utilização de factores.

${ }^{3}$ Um número índice é definido como um número real que mede alterações num conjunto de variáveis relacionadas. Conceptualmente, os números índices podem ser utilizados para comparações ao longo do tempo, no espaço ou para ambos.

${ }^{4}$ Esta questão tem particular relevância no caso português, cuja economia, em 1997, foi considerada a sétima mais globalizada a nível mundial (The Economist, April, 8th, 2000: 128).

${ }^{5}$ Estas propriedades são: positividade (o número índice é positivo); continuidade (o índice é uma função contínua dos preços e quantidades); proporcionalidade (a uma mudança proporcional em todos os preços ou quantidades corresponde uma alteração na mesma proporção do índice); invariância dimensional (o índice é invariante a mudanças na unidade de medida); teste reverso-temporal (para dois períodos, s e t, o índice no período t é igual ao inverso do do período s); teste valor-médio (o índice quantidade localiza-se entre as alterações mínima e máxima ao nível da quantidade produzida do bem); teste factor-reverso (se for utilizada a mesma fórmula para calculo dos índices preço e quantidade, o produto dos mesmos é igual ao rácio dos valores); teste de circularidade - transitividade (para quaisquer três períodos, s, t e r, tem-se o índice quantidade $\mathrm{Q}_{\mathrm{st}}=\mathrm{Q}_{\mathrm{sr}} \mathrm{xQ} \mathrm{Q}_{\mathrm{rt}}$, ou seja, a comparação directa entre s e t proporciona o mesmo 
índice que a comparação indirecta através de r). Destas propriedades, o teste de circularidade não é satisfeito pelo índice de Fischer, enquanto o índice de Törnqvist não preenche os testes factor-reverso e circularidade (Coelli et al., 1998: 80).

${ }^{6}$ Em vez de períodos de tempo, sem perda de generalidade, s e t também podem referir-se a empresas.

${ }^{7}$ Salienta-se, no entanto, que a teoria económica subjacente aos índices de Fischer e de Törnqvist assume que as empresas observadas no período s e t são produtivamente eficientes, tanto em termos técnicos como económicos, localizando-se na fronteira de produção, custo, lucro ou rendimento (Coelli et al., 1998: cap. 5).

8 "Superlativos" no sentido de que são exactos para uma forma funcional flexível. "Forma flexível" é a que proporciona uma aproximação de segunda ordem a uma forma funcional arbitrária duplamente diferenciável de modo contínuo. "Exacto" significa que se pode calcular um índice de produtividade não paramétrico que é "exactamente" compatível com a forma translogarítmica (Färe et al., 1994).

${ }^{9}$ Se as funções distância para os períodos s e t forem ambas representadas por funções translog com idênticos parâmetros de segunda ordem, então a média geométrica dos índices de Malmquist baseados nas tecnologias dos períodos s e t é equivalente ao índice quantidade de Törnqvist. Se as funções translog forem substituídas por funções quadráticas, a situação anterior ocorre para o índice de Fischer (Coelli, et al., 1998: cap. 5).

${ }^{10}$ Este é o método que, em aplicações empíricas, tem sido utilizado com mais frequência. Vejam-se, por exemplo, os trabalhos de Berg et al. (1992), Färe et al. (1995), Pastor (1995), Griffel-Tatjé e Lovell (1995), Rao e Coelli (1998), Rebelo e Mendes (2000).

${ }^{11}$ Estamos, no entanto, cientes que há excepções. É, por exemplo, o caso dos agricultores com quotas leiteiras, os quais tentam satisfazer a mesma com o mínimo consumo de factores.

${ }^{12}$ Uma hipótese alternativa à forte dispensabilidade é a "fraca dispensabilidade", a qual estabelece que se um vector de produtos (y) pode ser produzido com certo vector de factores (x) então qualquer contracção de y (?y), com $0<$ ? $<1$, é igualmente possível de se produzir com $\mathrm{x}$. Facilmente se verifica que a forte dispensabilidade implica a fraca dispensabilidade, não sendo o contrário verdadeiro.

${ }^{13}$ Uma vez que $\mathrm{x}^{*} \mathrm{e} \mathrm{x}$ são vectores, $\mathrm{x} * \mathrm{x}$ apenas é válido quando todos os elementos de $\mathrm{x}^{*}$ foram não inferiores aos correspondentes de $\mathrm{x}$.

${ }^{14}$ Para que o índice PTF de Malmquist satisfaça a propriedade da circularidade (descrita na nota de rodapé $n^{\circ} 5$ ) devem comparar-se períodos próximos. Em geral esta propriedade não é satisfeita quando se comparam períodos com grande desfasamento (Berg, et al., 1992). 
${ }^{15}$ Como a escolha da tecnologia de referência pode afectar os resultados, geralmente, considera-se o índice de Malmquist como a média geométrica dos índices correspondentes a cada uma das tecnologias de referência (Grifel-Tatjé e Lovell, 1996), ainda que estritamente não o seja.

${ }^{16}$ A última componente da expressão (5) indica o cálculo do índice PTF de Malmquist de acordo com a representação gráfica incluída na figura 2 infra.

${ }^{17}$ Malmquist (1953) introduziu o índice quantidade no consumo, definindo-o como o montante pelo qual uma combinação de bens consumidos deve ser radialmente multiplicada de modo a gerar o mesmo nível de utilidade que o proporcionado pela combinação base de bens. Uma vez que este índice foi inicialmente enquadrado na teoria do consumidor, com a utilidade a ser apenas ordinalmente medida, a noção de economias de escala é irrelevante, o que não sucede na teoria da produção.

${ }^{18}$ Apesar da PTF ser estimada para a situação de RCE, estes problemas podem ser resolvidos em situações de rendimentos variáveis à escala, de modo a decompor-se a eficiência técnica global (calculada sob RCE) nos termos eficiência técnica pura (determinada sob a hipótese de RVE) e eficiência técnica de escala. Esta é igual à eficiência técnica global dividida pela eficiência técnica pura.

${ }^{19}$ O software DEAP de Coelli (1996b) permite resolver de forma muito fácil estes problemas, gerando resultados finais que contêm os índices de eficiência técnica sob RCE e RVE, os índices PTF, AT, AE e ainda a decomposição de AE em alteração da eficiência técnica pura e alteração da eficiência de escala.

${ }^{20}$ Contrariamente às funções custo e lucro duais, a função de produção é aplicável apenas aos casos em que se considera a produção de um único produto.

${ }^{21}$ Os softwares LIMDEP de Greene (1998) e FRONTIER de Coelli (1996a) contêm rotinas específicas para a estimação deste tipo de modelos, incluindo o cômputo dos índices individuais de eficiência técnica.

${ }^{22}$ A expressão (14) é a utilizada por (Coelli, 1996a), sendo um refinamento da metodologia inicialmente proposta por Jondrow et al. (1982).

${ }^{23}$ No entanto, como referem Coelli et al.(1998: 234), "acreditamos que as medidas de alteração obtidas são muito mais facilmente obtidas pelo processo acima mencionado".

${ }^{24}$ Agradecemos à Direcção Regional de Agricultura de Trás-os-Montes do MADRP a pronta disponibilidade dos dados.

${ }^{25}$ Foi considerado um panel equilibrado para se poder estimar o "modelo ineficiência com variação no tempo" proposto por Battese e Coelli (1992), no qual $u_{i t}=\{\exp [-?(t-$ $\mathrm{T})]\} \mathrm{u}_{\mathrm{i}}, \mathrm{i}=1, \ldots, \mathrm{N}$ e $\mathrm{t}=1, \ldots, \mathrm{T}$. Este modelo foi, no entanto, rejeitado (? estatisticamente não significativo).

${ }^{26} \mathrm{O}$ processo de eliminação descrito levou à exclusão de 73 observações de um total de 281. 
${ }^{27}$ Se compararmos, em termos pontuais, a produtividade aparente de 1997 com a de 1990, os resultados são: SAU $+11,8 \%$; trabalho $+46,6 \%$; outros factores $+16,7 \%$; e capital $-38,8 \%$.

${ }^{28}$ Os resultados são invariantes à escolha do factor de normalização.

${ }^{29}$ A própria forma funcional translog é uma expansão de segunda ordem em série de Taylor à volta de um ponto arbitrário subjacente à "verdadeira tecnologia".

${ }^{30}$ No anexo 2, quadros 2.1 e 2.2, constam, para a totalidade das observações, os índices de eficiência técnica calculados pelos métodos DEA e AFE.

${ }^{31}$ Considerando as 208 observações, os coeficientes de correlação de Pearson e de Spearman são de 0,725 e de 0,655 (ambos estatisticamente significativos ao nível de 1\%), valores que apontam para uma clara consistência entre os índices de ET calculados por ambos os métodos. 
Medição da evolução da produtividade total dos factores: o índice de Malmquist

\section{Anexo 1}

ESTIMATIVAS DOS PARÂMETROS DO MODELO

\section{FRONTEIRA ESTOCÁSTICO}

Quadro 1

MODELO FRONTEIRA ESTOCÁSTICO

\begin{tabular}{ccc}
\hline Parâmetro & Estimativa & Rácio t \\
\hline$?_{0}$ & 0,2536 & 5,08 \\
$?_{\mathrm{S}}$ & 0,2011 & 2,75 \\
$?_{\mathrm{L}}$ & $0,1750^{*}$ & \\
$?_{\mathrm{K}}$ & 0,0155 & 0,71 \\
$?_{\mathrm{F}}$ & 0,6084 & 18,59 \\
$?_{\mathrm{SS}}$ & $-0,1307$ & $-0,41$ \\
$?_{\mathrm{LL}}$ & $-0,1755^{*}$ & \\
$?_{\mathrm{KK}}$ & $-0,0087$ & $-0,38$ \\
$?_{\mathrm{FF}}$ & $0,1749^{*}$ & 2,17 \\
$?_{\mathrm{SL}}$ & 0,1448 & \\
$?_{\mathrm{SK}}$ & $-0,0255$ & $-0,41$ \\
$?_{\mathrm{SF}}$ & 0,0109 & 0,09 \\
$?_{\mathrm{LK}}$ & $0,0416^{*}$ & \\
$?_{\mathrm{LF}}$ & $-0,0109$ & $-0,09$ \\
$?_{\mathrm{KF}}$ & $-0,0074$ & $-0,25$ \\
$?_{\mathrm{t}}$ & 0,0177 & 1,84 \\
$?_{\mathrm{tt}}$ & 0,0111 & 1,19 \\
$?_{\mathrm{St}}$ & $-0,0252$ & 0,75 \\
$?_{\mathrm{Lt}}$ & $0,0171^{*}$ & \\
$?_{\mathrm{Kt}}$ & $-0,0033$ & $-0,44$ \\
$?_{\mathrm{Ft}}$ & 0,0114 & 0,74 \\
$?$ & 0,8159 & 12,06 \\
\hline
\end{tabular}

* Valores calculados considerando as expressões expressas por (19). 


\section{Anexo 2 \\ ÍNDICES DE EFICIÊNCIA TÉCNICA}

Quadro 2.1

EFICIÊNCIA TÉCNICA: MÉTODO DEA

\begin{tabular}{cccccccccc}
\hline & 1990 & 1991 & 1992 & 1993 & 1994 & 1995 & 1996 & 1997 & M. Geom. \\
\hline 1 & 0,422 & 0,910 & 0,463 & 1,000 & 0,594 & 1,000 & 0,795 & 0,748 & 0,708 \\
2 & 1,000 & 0,888 & 0,493 & 0,668 & 0,906 & 0,530 & 0,831 & 0,824 & 0,746 \\
3 & 0,612 & 0,850 & 1,000 & 0,973 & 0,687 & 1,000 & 1,000 & 1,000 & 0,876 \\
4 & 0,375 & 0,661 & 1,000 & 1,000 & 0,713 & 1,000 & 1,000 & 1,000 & 0,805 \\
5 & 1,000 & 1,000 & 0,420 & 0,770 & 0,950 & 0,845 & 0,909 & 0,875 & 0,821 \\
6 & 0,618 & 0,844 & 0,915 & 1,000 & 1,000 & 1,000 & 1,000 & 0,558 & 0,848 \\
7 & 1,000 & 1,000 & 1,000 & 0,283 & 0,930 & 0,974 & 0,891 & 1,000 & 0,831 \\
8 & 0,775 & 1,000 & 0,863 & 0,817 & 0,967 & 1,000 & 0,897 & 1,000 & 0,911 \\
9 & 1,000 & 1,000 & 1,000 & 1,000 & 1,000 & 1,000 & 1,000 & 1,000 & 1,000 \\
10 & 0,912 & 0,732 & 0,856 & 0,728 & 0,588 & 0,494 & 0,576 & 0,680 & 0,683 \\
11 & 0,537 & 0,709 & 0,410 & 0,734 & 0,851 & 0,293 & 0,634 & 0,365 & 0,534 \\
12 & 0,637 & 1,000 & 1,000 & 1,000 & 0,606 & 0,687 & 1,000 & 1,000 & 0,847 \\
13 & 1,000 & 0,877 & 0,521 & 0,745 & 0,823 & 0,738 & 0,763 & 0,628 & 0,749 \\
14 & 0,768 & 0,435 & 0,236 & 0,521 & 0,620 & 0,653 & 0,604 & 0,433 & 0,507 \\
15 & 1,000 & 0,823 & 0,837 & 1,000 & 1,000 & 0,483 & 1,000 & 0,759 & 0,842 \\
16 & 1,000 & 0,835 & 0,864 & 0,771 & 1,000 & 1,000 & 0,550 & 1,000 & 0,862 \\
17 & 0,840 & 0,940 & 0,482 & 0,616 & 0,501 & 1,000 & 0,797 & 0,569 & 0,693 \\
18 & 0,344 & 0,465 & 0,450 & 0,792 & 0,533 & 0,807 & 0,542 & 0,205 & 0,478 \\
19 & 0,898 & 0,773 & 0,328 & 0,502 & 0,691 & 0,827 & 0,861 & 0,329 & 0,607 \\
20 & 0,584 & 0,658 & 0,509 & 0,707 & 0,670 & 0,892 & 1,000 & 0,501 & 0,672 \\
21 & 0,850 & 0,816 & 0,769 & 0,863 & 0,545 & 0,891 & 0,978 & 0,834 & 0,808 \\
22 & 0,870 & 0,847 & 0,410 & 1,000 & 0,472 & 1,000 & 0,689 & 0,781 & 0,725 \\
23 & 0,972 & 0,802 & 0,533 & 0,669 & 0,906 & 0,811 & 0,864 & 0,759 & 0,778 \\
24 & 0,952 & 0,362 & 0,339 & 0,558 & 1,000 & 0,987 & 0,730 & 0,404 & 0,609 \\
25 & 1,000 & 1,000 & 1,000 & 0,738 & 1,000 & 0,960 & 1,000 & 0,968 & 0,954 \\
26 & 0,814 & 1,000 & 0,615 & 0,667 & 1,000 & 1,000 & 0,910 & 1,000 & 0,862 \\
\hline & 0,765 & 0,792 & 0,613 & 0,747 & 0,767 & 0,810 & 0,823 & 0,687 & 0,747 \\
\hline
\end{tabular}


Medição da evolução da produtividade total dos factores: o índice de Malmquist

\section{Quadro 2.2}

EFICIÊNCIA TÉCNICA (ET): MÉTODO AFE

\begin{tabular}{cccccccccc}
\hline & 1990 & 1991 & 1992 & 1993 & 1994 & 1995 & 1996 & 1997 & M. Geom. \\
\hline 1 & 0,553 & 0,824 & 0,624 & 0,851 & 0,686 & 0,891 & 0,821 & 0,858 & 0,754 \\
2 & 0,796 & 0,781 & 0,764 & 0,736 & 0,859 & 0,562 & 0,848 & 0,875 & 0,771 \\
3 & 0,639 & 0,806 & 0,823 & 0,823 & 0,779 & 0,763 & 0,887 & 0,907 & 0,799 \\
4 & 0,478 & 0,659 & 0,898 & 0,923 & 0,428 & 0,812 & 0,829 & 0,837 & 0,708 \\
5 & 0,814 & 0,897 & 0,742 & 0,839 & 0,871 & 0,770 & 0,872 & 0,635 & 0,801 \\
6 & 0,756 & 0,763 & 0,748 & 0,870 & 0,868 & 0,735 & 0,798 & 0,529 & 0,751 \\
7 & 0,731 & 0,790 & 0,953 & 0,415 & 0,811 & 0,735 & 0,748 & 0,817 & 0,733 \\
8 & 0,855 & 0,881 & 0,758 & 0,824 & 0,874 & 0,821 & 0,852 & 0,897 & 0,844 \\
9 & 0,807 & 0,863 & 0,742 & 0,769 & 0,862 & 0,426 & 0,752 & 0,835 & 0,742 \\
10 & 0,845 & 0,721 & 0,900 & 0,845 & 0,629 & 0,509 & 0,751 & 0,778 & 0,737 \\
11 & 0,618 & 0,708 & 0,593 & 0,726 & 0,844 & 0,367 & 0,710 & 0,550 & 0,623 \\
12 & 0,712 & 0,936 & 0,859 & 0,822 & 0,709 & 0,685 & 0,873 & 0,932 & 0,810 \\
13 & 0,816 & 0,827 & 0,831 & 0,840 & 0,833 & 0,708 & 0,797 & 0,743 & 0,798 \\
14 & 0,770 & 0,640 & 0,350 & 0,591 & 0,731 & 0,628 & 0,681 & 0,613 & 0,611 \\
15 & 0,879 & 0,823 & 0,773 & 0,728 & 0,908 & 0,426 & 0,883 & 0,797 & 0,760 \\
16 & 0,921 & 0,799 & 0,823 & 0,745 & 0,916 & 0,628 & 0,481 & 0,792 & 0,749 \\
17 & 0,859 & 0,909 & 0,763 & 0,765 & 0,602 & 0,867 & 0,817 & 0,707 & 0,780 \\
18 & 0,466 & 0,536 & 0,584 & 0,677 & 0,663 & 0,647 & 0,631 & 0,349 & 0,557 \\
19 & 0,832 & 0,807 & 0,588 & 0,641 & 0,698 & 0,725 & 0,839 & 0,402 & 0,675 \\
20 & 0,681 & 0,728 & 0,787 & 0,783 & 0,786 & 0,805 & 0,918 & 0,625 & 0,760 \\
21 & 0,756 & 0,820 & 0,895 & 0,802 & 0,679 & 0,782 & 0,866 & 0,878 & 0,807 \\
22 & 0,826 & 0,820 & 0,613 & 0,817 & 0,594 & 0,840 & 0,681 & 0,826 & 0,745 \\
23 & 0,744 & 0,796 & 0,847 & 0,754 & 0,843 & 0,788 & 0,838 & 0,727 & 0,791 \\
24 & 0,868 & 0,481 & 0,559 & 0,669 & 0,872 & 0,856 & 0,807 & 0,527 & 0,687 \\
25 & 0,556 & 0,627 & 0,773 & 0,784 & 0,742 & 0,559 & 0,761 & 0,679 & 0,679 \\
26 & 0,669 & 0,816 & 0,862 & 0,832 & 0,884 & 0,843 & 0,836 & 0,895 & 0,827 \\
\hline Geom. & 0,729 & 0,763 & 0,734 & 0,756 & 0,758 & 0,682 & 0,785 & 0,711 & 0,739 \\
\hline
\end{tabular}




\section{REFERÊNCIAS BIBLIOGRÁFICAS}

AIGNER, D. J.; LOVELL, C. A. K. e SCHMIDT, P. (1977), 'Formulation and Estimation of Stochastic Frontier Production Function Models". Journal of Econometrics, 6: 21-37.

BATTESE, G. E. e COELLI T. J. (1988), "Prediction of Firm Level Technical Efficiency with a Generalized Frontier Production Function and Panel Data", Journal of Econometrics, 38: 387-399.

BATTESE, G. E. e COELLI T. J. (1992), “ Frontier Production Functions, Technical Efficiency and Panel Data: With Application to Paddy Farmers in India", Journal of Productivity Analysis, 3: 153-169.

BERG, S.; FORSUND, F. e JANSEN, E. (1992), "Malmquist Indices of Productivity Growth During the Deregulation of Norwegian Banking 1980-89”, Scandinavian Journal of Economics, 94, Supplement: 211-228

BOSKIN, Michael J.; DULBERGER, E. R.; GORDON, R. J.; GRILICHES, Z. e JORGENSON, D. W. (1997). "Applied Economics in Action: The CPI Commission - Findings and Recommendations", American Economic Review, 87(2): 78-83.

COELLI, T. (1996a), "A Guide to FRONTIER Version 4.1: A Computer Program for Frontier Production Function Estimation”, CEPA Working Paper 96/07, Armidale Department of Econometrics, University of New England.

COELLI, T. (1996b). "A Guide to DEAP Version 2.1: A Data Envelopment Analysis (Computer) Program", CEPA Working Paper 96/08, Armidale Department of Econometrics, University of New England.

COELlI, T.; RAO, D. S. e BATTESE, G. E. (1998), An Introduction to Efficiency and Productivity Analysis, Boston, Kluwer Academic Publishers.

FÄRE, R.; GROSSKOPF, S.; MORRIS, M. e ZHANG, Z. (1994), "Productivity Growth, Technical Progress and Efficiency Changes in Industrialised Countries", American Economic Review, 84: 66-83.

FÄRE, R.; GROSSKOPF, S., LINDGREN, P. e ROOS, P. (1995), "Productivity Developments in Swedish Hospitals: A Malmquist Produto Index Approach", in: A. Charnes, W. W. Cooper, A. Y. Lewin and L. M. Seiford, Eds., Data Envelopment Analysis: Theory, Methodology and Applications. Kluwer Academic Publishers, Dordrecht.

FARREL, M. J. (1957), "The Measurement of Productive Efficiency", Journal of the Royal Statistical Society, Series A, 120, 253-281.

FISHER, I. (1922), The Making of Index Numbers. Boston, Hougton-Miflin. 
Medição da evolução da produtividade total dos factores: o índice de Malmquist

FORSUND, F. R.; LOVELL, C. A. K. e SCHMIDT, P. (1980), “A Survey of Frontier Productions and of their Relationship to Efficiency Measurement", Journal of Econometrics, 13: 5-25.

GREENE, William, H. (1998), LIMDEP, Version 7.0 User's Manual, Revised Edition, New York. Econometric Software, Inc.

GRIFELL-TATJÉ, E. e LOVELL, C. A. K. (1995), "A Note on the Malmquist Productivity Index”, Economic Letters, 47: 169-175.

GRIFELL-TATJÉ, E. e LOVELL, C. A. K. (1996), "Deregulation and Productivity Decline: The Case of Spanish Saving Banks", European Economic Review 40, 1281-1303.

JONDROW, J.; LOVELL C. A.; MATEROV, I. S. e SCHMIDT, P. (1982), “On Estimation of Technical Inefficiency in the Stochastic Frontier Production Function Model", Journal of Econometrics, 19: 233-238.

MALMQUIST, S. (1953), "Index Numbers and Indifference Curves", Trabajos de Estatistica 4(1), 209-242.

MEEUSEN, W. e van den Broeck (1977), Efficiency Estimation from Cobb-Douglas Production Functions with Composed Error", International Economic Review, 18: 435-444.

PASTOR, J. M. (1995), "Eficiencia, Cambio Productivo y Cambio Técnico en los Bancos y Cajas de Ahorro Espanolas: Uma Analisys Fronteira no Paramétrico”. Working Paper-EC 95-09. Valencia, Instituto Valenciano de Investigaciones Economicas.

PORTER, Michael (1990), The Competitive Advantage of Nations. London, The Macmillan Press.

RAO, D. S. e COELLI, T. J. (1998), "A Cross-Country Analysis of GDP Growth, Catchup and Convergence in Productivity, an Inequality", Paper presented at the 25th General Conference of the International Association for Research in Income and Wealth. Cambridge, England, August 1998.

REBELO, João (1992), Análise de Relações Custo-Produção e Eficiência Produtiva em Empresas Multiproduto: O Caso das Adegas Cooperativas da Região Demarcada do Douro (Tese de Doutoramento, UTAD, Vila Real, pol.).

REBELO, J. e MENDES, V. (2000), "Malmquist Indices of Productivity Change in Portuguese Banking: The Deregulation Period", International Advances in Economic Research, Vol. VI(3), August : 531-543

REBELO, J. e MENDES, V. (1997), "Progresso Tecnológico no Sector Bancário Português: 1990 - 95”, Revista da Banca, 42: 5-22.

The Economist (1996), The Unmeasurable Lightness of Being, The Economist, November 23rd: 85-86.

The Economist (2000), Emerging - Market Indicators, The Economist, April 8th: 128.

TÖRNQVIST, L. (1936), “The Bank of Finland's Consumption Price Index”, Bank of Finland Monthly Bulletin 10: 1-8. 